\title{
Synthesis of Free-base 10-Azacorroles
}

\section{Hiroto Omori, Satoru Hiroto, ${ }^{\star}$ and Hiroshi Shinokubo*}

Department of Applied Chemistry, Graduate School of Engineering, Nagoya University, Chikusa-ku, Nagoya 464-8603, Japan.

\section{E-mail: hshino@apchem.nagoya-u.ac.jp; hiroto@apchem.nagoya-u.ac.jp}

Table of Contents

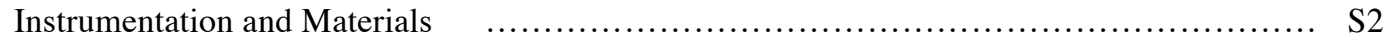

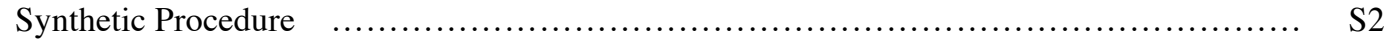

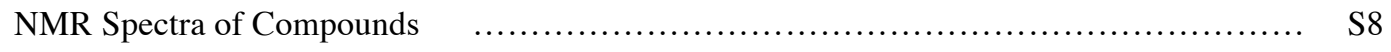

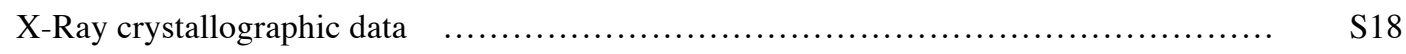

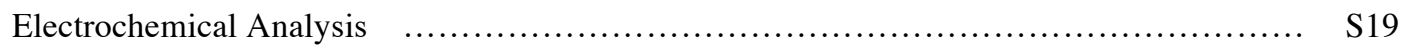

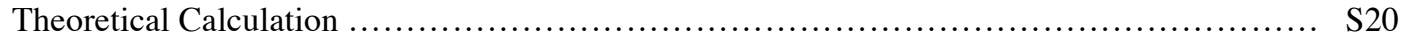




\section{Instrumentation and Materials}

${ }^{1} \mathrm{H}$ NMR $(500 \mathrm{MHz})$ and ${ }^{13} \mathrm{C} \mathrm{NMR}(126 \mathrm{MHz})$ spectra were recorded on a Bruker AVANCE III HD spectrometer, and chemical shifts were reported as the delta scale in ppm relative to $\mathrm{CHCl}_{3}(\delta=7.260 \mathrm{ppm})$, acetone- $d_{5}(\delta=2.050 \mathrm{ppm})$ for ${ }^{1} \mathrm{H} \mathrm{NMR}$ and $\mathrm{CDCl}_{3}(\delta=77.16 \mathrm{ppm})$, acetone- $d_{6}(\delta=29.84 \mathrm{ppm})$ for ${ }^{13} \mathrm{C}$ NMR. UV/vis/NIR absorption spectra were recorded on a Shimadzu UV-2550 or JASCO V670 spectrometer. Emission spectra were recorded using a JASCO FP-6500 spectrometer, and absolute fluorescence quantum yields were measured by the photon-counting method using an integration sphere. High-resolution mass spectra were recorded on a Bruker microTOF using positive mode ESI-TOF method for acetonitrile solutions. Unless otherwise noted, materials obtained from commercial suppliers were used without further purification.

\section{X-ray diffraction analysis}

X-ray data were obtained using a Bruker D8 QUEST X-ray diffractometer with an $\mathrm{I} \mu \mathrm{S}$ microfocus X-ray source and a large area $(10 \mathrm{~cm} \times 10 \mathrm{~cm})$ CMOS detector (PHOTON 100) for 2a, 3a, and 5a. Crystallographic details are given in CIF files. Fine crystals of $\mathbf{2 a}$ and 3a for the X-ray diffraction analysis were obtained by the vapor diffusion of methanol into its chloroform solution. For the X-ray crystal structure of 5a, a fine crystal for the X-ray diffraction analysis was obtained by the vapor diffusion of methanol into its 1,2-dichloroethane solution. The detailed crystallographic data for all compounds are listed in Table S1. Crystallographic data for $\mathbf{2 a}, \mathbf{3 a}$, and 5a have been deposited with the Cambridge Crystallographic Data Centre as supplementary publication no. CCDC-1477606, 1477608, and 1477610, respectively.

\section{Synthesis and Compound Data}

\section{meso-3,5-Dimethoxyphenyl- $\alpha, \alpha$ '-dibromodipyrrin.}

A two-necked flask containing meso-3,5-dimethoxyphenyldipyrromethane (424 $\mathrm{mg}, 1.50$ mmol) was evacuated and then refilled with $\mathrm{N}_{2}$. To the flask, dry THF $(22.5 \mathrm{~mL})$ was added and cool to $-78{ }^{\circ} \mathrm{C}$. NBS (534 mg, $3.00 \mathrm{mmol}$ ) was added to the solution in three portions every $15 \mathrm{~min}$. After complete addition of NBS, the solution was stirred for $1 \mathrm{~h}$. Then DDQ (340 mg, $1.50 \mathrm{mmol})$ was added and stirred at $-78^{\circ} \mathrm{C}$ for 10 min and at room temperature for $20 \mathrm{~min}$. The reaction mixture was subjected to alumina column chromatography $\left(\mathrm{CH}_{2} \mathrm{Cl}_{2}\right.$ as an eluent) and evaporated. Purification by silica-gel column chromatography with $\mathrm{CH}_{2} \mathrm{Cl}_{2} /$ hexane afforded the title compound in $83 \%(547 \mathrm{mg}, 1.25 \mathrm{mmol})$ as an orange solid. ${ }^{1} \mathrm{H} \mathrm{NMR}$ $\left(\mathrm{CDCl}_{3}\right): \delta 12.4($ br s, $1 \mathrm{H}, \mathrm{NH}), 6.57-6.59(\mathrm{~m}, 5 \mathrm{H}), 6.33(\mathrm{~d}, J=4.0 \mathrm{~Hz}, 2 \mathrm{H}$, pyrrole- $\beta$ ), $3.81(\mathrm{~s}, 6 \mathrm{H}, \mathrm{OMe})$ 
ppm; ${ }^{13} \mathrm{C} \mathrm{NMR}\left(\mathrm{CDCl}_{3}\right): \delta 160.2,140.2,139.2,137.3,130.2,129.7,120.5,109.3,101.4,55.6$ ppm; HR-MS (ESI-MS): $m / z=438.9467$, calcd for $\left(\mathrm{C}_{17} \mathrm{H}_{15} \mathrm{~N}_{2} \mathrm{O}_{2} \mathrm{Br}_{2}\right)^{+}=438.9475\left[(\mathrm{M}+\mathrm{H})^{+}\right]$.

\section{$N, N$-Bis(meso-phenyl-1-bromodipyrrin-9-yl)- $N$-benzylamine.}

A Schlenk tube containing meso-phenyl- $\alpha, \alpha^{\prime}$-dibromodipyrrin (152 mg, $\left.402 \mu \mathrm{mol}\right), \mathrm{Pd}_{2}(\mathrm{dba})_{3}{ }^{\bullet} \mathrm{CHCl}_{3}(10.7$ $\mathrm{mg}, 10.4 \mu \mathrm{mol})$, Xantphos (13.0 mg, $22.5 \mu \mathrm{mol})$, and $\mathrm{KO}^{t} \mathrm{Bu}(113.1 \mathrm{mg}, 1.01 \mathrm{mmol})$ was evacuated and then refilled with $\mathrm{N}_{2}$. To the tube, benzylamine $(37.0 \mu \mathrm{L}, 340 \mu \mathrm{mol})$, and dry and degassed toluene $(5.20 \mathrm{~mL})$ were added. The mixture was stirred at $80{ }^{\circ} \mathrm{C}$ for $13 \mathrm{~h}$. The reaction mixture was passed through a short plug of silica $\left(\mathrm{CHCl}_{3}\right.$ as an eluent) and evaporated. Purification by silica-gel column chromatography with $\mathrm{CHCl}_{3} /$ hexane afforded the title compound in $53 \%(74.9 \mathrm{mg}, 107 \mu \mathrm{mol})$ as a black solid. ${ }^{1} \mathrm{H}$ NMR (acetone- $d_{6}$ ): $\delta 12.9$ (br s, 2H, NH), 7.55 (d, $J=7.5 \mathrm{~Hz}, 2 \mathrm{H}, \mathrm{Bn}-\mathrm{Ph}$ ), 7.47-7.52 (m, 10H, meso-Ph), 7.42 (dd, $\left.J^{l}=J^{2}=7.8 \mathrm{~Hz}, 2 \mathrm{H}, \mathrm{Bn}-\mathrm{Ph}\right), 7.30(\mathrm{t}, J=7.3 \mathrm{~Hz}, 1 \mathrm{H}, \mathrm{Bn}-\mathrm{Ph}), 6.98(\mathrm{~d}, J=5.0 \mathrm{~Hz}, 2 \mathrm{H}$, pyrrole- $\beta), 6.84(\mathrm{~d}, J=$ $4.5 \mathrm{~Hz}, 2 \mathrm{H}$, pyrrole- $\beta$ ), 6.23 (d, $J=4.0 \mathrm{~Hz}, 2 \mathrm{H}$, pyrrole- $\beta$ ), 6.13 (d, $J=4.0 \mathrm{~Hz}, 2 \mathrm{H}$, pyrrole- $\beta$ ), 5.59 (s, $2 \mathrm{H}$, $\left.\mathrm{CH}_{2}\right) \mathrm{ppm} ;{ }^{13} \mathrm{C}$ NMR $\left(\mathrm{CDCl}_{3}\right): \delta 163.0,145.0,137.8,136.7,136.6,135.2,133.9,131.0,129.1,128.7,127.9$, $127.5,126.7,120.7,118.3,113.7,109.9,53.5 \mathrm{ppm}$; HR-MS (ESI-MS): $\mathrm{m} / \mathrm{z}=702.0713$, calcd for $\left(\mathrm{C}_{37} \mathrm{H}_{28} \mathrm{~N}_{5} \mathrm{Br}_{2}\right)^{+}=702.0689\left[(\mathrm{M}+\mathrm{H})^{+}\right]$.

\section{$N, N$-Bis(meso-phenyl-1-bromodipyrrin-9-yl)- $N$-benzylamine Zn(II) complex 1 b.}

A flask containing $N, N$-bis(meso-phenyl-1-bromodipyrrin-9-yl)- $N$-benzylamine (44.0 mg, $62.7 \mu \mathrm{mol}$ ), $\mathrm{NaOAc}(25.2 \mathrm{mg}, 307 \mu \mathrm{mol})$, and $\mathrm{Zn}(\mathrm{OAc})_{2} \bullet 2 \mathrm{H}_{2} \mathrm{O}(68.2 \mathrm{mg}, 311 \mu \mathrm{mol})$ was charged with $\mathrm{MeOH}(20 \mathrm{~mL})$. The mixture was stirred at reflux for $3 \mathrm{~h}$. The reaction mixture was extracted with EtOAc. The organic layer was washed with water, dried over anhydrous $\mathrm{Na}_{2} \mathrm{SO}_{4}$, and concentrated in vacuo. Purification by silica-gel column chromatography with EtOAc/hexane afforded the title compound in $80 \%(38.3 \mathrm{mg}, 50.1 \mu \mathrm{mol})$ as a green solid. ${ }^{1} \mathrm{H}$ NMR (acetone- $d_{6}$ ): $\delta 7.48$ (br s, $10 \mathrm{H}$, meso- $\mathrm{Ph}$ ), 7.24-7.35 (m, 5H, Bn- $\mathrm{Ph}$ ), 6.68 (d, $J=4.5 \mathrm{~Hz}$, $2 \mathrm{H}$, pyrrole- $\beta$ ), 6.54 (d, $J=4.5 \mathrm{~Hz}, 2 \mathrm{H}$, pyrrole- $\beta$ ), 6.42 (d, $J=4.0 \mathrm{~Hz}, 2 \mathrm{H}$, pyrrole $-\beta), 6.31$ (d, $J=3.5 \mathrm{~Hz}$, $2 \mathrm{H}$, pyrrole- $\beta$ ), $5.49\left(\mathrm{~d}, J=18 \mathrm{~Hz}, 1 \mathrm{H}, \mathrm{CH}_{2}\right), 5.31\left(\mathrm{~d}, J=18 \mathrm{~Hz}, 1 \mathrm{H}, \mathrm{CH}_{2}\right) \mathrm{ppm} ;{ }^{13} \mathrm{C}$ NMR (acetone- $\left.d_{6}\right): \delta$ 159.6, 142.2, 140.9, 140.2, 139.1, 138.2, 135.7, 131.6, 129.7, 129.4, 129.2, 128.7, 128.4, 128.3, 126.9, 118.5, 112.8, 54.3 ppm; HR-MS (ESI-MS): $m / z=765.9783$, calcd for $\left(\mathrm{C}_{37} \mathrm{H}_{26} \mathrm{~N}_{5} \mathrm{Br}_{2} \mathrm{Zn}\right)^{+}=765.9792\left[(\mathrm{M}+\mathrm{H})^{+}\right]$.

\section{$N, N$-Bis(meso-3,5-dimethoxyphenyl-1-bromodipyrrin-9-yl)- $N$-benzylamine.}


A Schlenk tube containing meso-3,5-dimethoxyphenyl- $\alpha, \alpha$-dibromodipyrrin (263 mg, $600 \mu \mathrm{mol})$, $\mathrm{Pd}_{2}(\mathrm{dba})_{3}{ }^{\bullet} \mathrm{CHCl}_{3}(15.6 \mathrm{mg}, 15.1 \mu \mathrm{mol})$, Xantphos $(19.2 \mathrm{mg}, 33.2 \mu \mathrm{mol})$, and $\mathrm{KO}{ }^{t} \mathrm{Bu}(169 \mathrm{mg}, 1.51 \mathrm{mmol})$ was evacuated and then refilled with $\mathrm{N}_{2}$. To the tube, benzylamine $(60.0 \mu \mathrm{L}, 552 \mu \mathrm{mol})$ and dry and degassed toluene $(9.00 \mathrm{~mL})$ were added. The mixture was stirred at $80{ }^{\circ} \mathrm{C}$ for $11 \mathrm{~h}$. The reaction mixture was passed through a short plug of silica $\left(\mathrm{CHCl}_{3}\right.$ as an eluent) and evaporated. Purification by silica-gel column chromatography with $\mathrm{CHCl}_{3} /$ hexane afforded the title compound in $50 \%(123 \mathrm{mg}, 150 \mu \mathrm{mol})$ as a black solid. ${ }^{1} \mathrm{H}$ NMR (acetone- $\left.d_{6}\right)$ : $\delta 12.9(\mathrm{br} \mathrm{s}, 2 \mathrm{H}, \mathrm{NH}), 7.55(\mathrm{~d}, J=7.5 \mathrm{~Hz}, 2 \mathrm{H}, \mathrm{Ph}), 7.42\left(\mathrm{dd}, J^{l}=J^{2}=7.8 \mathrm{~Hz}, 2 \mathrm{H}, \mathrm{Ph}\right)$, $7.30(\mathrm{t}, J=7.5 \mathrm{~Hz}, 1 \mathrm{H}, \mathrm{Ph}), 6.99(\mathrm{~d}, J=5.0 \mathrm{~Hz}, 2 \mathrm{H}$, pyrrole- $\beta$ ), 6.96 (d, $J=5.0 \mathrm{~Hz}, 2 \mathrm{H}$, pyrrole- $\beta$ ), 6.61-6.63 $(\mathrm{m}, 6 \mathrm{H}, \mathrm{Ar}), 6.24(\mathrm{~d}, J=4.0 \mathrm{~Hz}, 2 \mathrm{H}$, pyrrole- $\beta), 6.22(\mathrm{~d}, J=4.0 \mathrm{~Hz}, 2 \mathrm{H}$, pyrrole- $\beta), 5.59\left(\mathrm{~s}, 2 \mathrm{H}, \mathrm{CH}_{2}\right) 3.83(\mathrm{~s}$, 12H, OMe) ppm; ${ }^{13} \mathrm{C}$ NMR (acetone- $d_{6}$ ): $\delta$ 163.5, 161.3, 145.1, 139.0, 139.0, 136.9, 136.1, 134.5, 129.8, 128.1, 127.4, 121.6, 118.9, 114.8, 110.9, 109.9, 101.5, 55.8, 53.8 ppm; HR-MS (ESI-MS): $\mathrm{m} / \mathrm{z}=822.1126$, calcd for $\left(\mathrm{C}_{41} \mathrm{H}_{36} \mathrm{~N}_{5} \mathrm{O}_{4} \mathrm{Br}_{2}\right)^{+}=822.1113\left[(\mathrm{M}+\mathrm{H})^{+}\right]$.

\section{$N, N$-Bis(meso-3,5-dimethoxyphenyl-1-bromodipyrrin-9-yl)- $N$-benzylamine $Z$ n(II) complex 1c.}

A flask containing $N, N$-bis(meso-3,5-dimethoxyphenyl-1-bromodipyrrin-9-yl)- $N$-benzylamine (49.1 mg, 59.8 $\mu \mathrm{mol}), \mathrm{NaOAc}(25.2 \mathrm{mg}, 307 \mu \mathrm{mol})$, and $\mathrm{Zn}(\mathrm{OAc})_{2} \cdot 2 \mathrm{H}_{2} \mathrm{O}(66.5 \mathrm{mg}, 303 \mu \mathrm{mol})$ was charged with $\mathrm{MeOH}(20$ $\mathrm{mL}$ ). The mixture was stirred at reflux for $6 \mathrm{~h}$. The reaction mixture was extracted with EtOAc. The organic layer was washed with water, dried over anhydrous $\mathrm{Na}_{2} \mathrm{SO}_{4}$, and concentrated in vacuo. Purification by silica-gel column chromatography with EtOAc/hexane afforded the title compound in $92 \%$ (48.9 mg, 55.3 $\mu \mathrm{mol})$ as a green solid. ${ }^{1} \mathrm{H}$ NMR (acetone- $\left.d_{6}\right): \delta 7.27-7.38(\mathrm{~m}, 5 \mathrm{H}, \mathrm{Ph}), 6.82(\mathrm{~d}, J=4.5 \mathrm{~Hz}, 2 \mathrm{H}$, pyrrole $-\beta)$, 6.62-6.67 (m, 6H, Ar), $6.58(\mathrm{~d}, J=4.5 \mathrm{~Hz}, 2 \mathrm{H}$, pyrrole- $\beta$ ), $6.45(\mathrm{~d}, J=4.0 \mathrm{~Hz}, 2 \mathrm{H}$, pyrrole- $\beta$ ), $6.42(\mathrm{~d}, J=4.5$ $\mathrm{Hz}, 2 \mathrm{H}$, pyrrole- $\beta$ ), $5.55\left(\mathrm{~d}, J=18 \mathrm{~Hz}, 1 \mathrm{H}, \mathrm{CH}_{2}\right), 5.38\left(\mathrm{~d}, J=18 \mathrm{~Hz}, 1 \mathrm{H}, \mathrm{CH}_{2}\right), 3.84(\mathrm{~s}, 12 \mathrm{H}, \mathrm{OMe}) \mathrm{ppm} ;{ }^{13} \mathrm{C}$ NMR (acetone- $\left.d_{6}\right): \delta 161.0,160.9,159.5,141.8,140.9,140.6,139.9,138.2,135.7,129.7,129.4,128.7,128.4$, 126.9, 118.5, 112.7, 110.2, 101.0, 55.8, $54.3 \mathrm{ppm}$; HR-MS (ESI-MS): $\mathrm{m} / \mathrm{z}=886.0188$, calcd for $\left(\mathrm{C}_{41} \mathrm{H}_{34} \mathrm{~N}_{5} \mathrm{O}_{4} \mathrm{Br}_{2} \mathrm{Zn}\right)^{+}=886.0215\left[(\mathrm{M}+\mathrm{H})^{+}\right]$.

\section{$N$-Benzyl-5,15-dimesityl-10-azacorrole $\mathrm{Zn}(\mathrm{II})$ complex $2 \mathrm{a}$.}

A Schlenk tube containing $N, N$-bis(meso-mesityl-1-bromodipyrrin-9-yl)- $N$-benzylamine $\mathrm{Zn}(\mathrm{II})$ complex (34.3 $\mathrm{mg}, 40.4 \mu \mathrm{mol}), 2,2$ '-bipyridyl $(11.9 \mathrm{mg}, 76.2 \mu \mathrm{mol})$, and $\mathrm{Ni}(\mathrm{cod})_{2}(23.1 \mathrm{mg}, 84.0 \mu \mathrm{mol})$ was evacuated and then refilled with $\mathrm{N}_{2}$. To the tube, 1,5-cyclooctadiene $(9.00 \mu \mathrm{L}, 85.8 \mu \mathrm{mol})$ and dry DMF $(4.00 \mathrm{~mL})$ were 
added. The mixture was stirred at $50{ }^{\circ} \mathrm{C}$ for $1 \mathrm{~h}$. The reaction mixture was extracted with EtOAc. The organic layer was washed with water, dried over anhydrous $\mathrm{Na}_{2} \mathrm{SO}_{4}$, and concentrated in vacuo. Purification by silica-gel column chromatography with EtOAc/hexane afforded the title compound in $83 \%(23.1 \mathrm{mg}, 33.5$ $\mu \mathrm{mol}$ ) as a purple solid. ${ }^{1} \mathrm{H}$ NMR (acetone- $\left.d_{6}\right)$ of $\mathbf{2 a :} \delta 8.00(\mathrm{~d}, J=3.5 \mathrm{~Hz}, 2 \mathrm{H}$, pyrrole- $\beta$ ), $7.94(\mathrm{~d}, J=4.5 \mathrm{~Hz}$, $2 \mathrm{H}$, pyrrole- $\beta$ ), 7.83 (d, $J=4.0 \mathrm{~Hz}, 2 \mathrm{H}$, pyrrole- $\beta$ ), 7.62 (d, $J=3.5 \mathrm{~Hz}, 2 \mathrm{H}$, pyrrole- $\beta$ ), 7.15-7.22 (m, 9H), $7.01\left(\mathrm{~s}, 2 \mathrm{H}, \mathrm{CH}_{2}\right), 2.49$ (s, 6H, $p$-Me), 1.91 (s, 12H,o-Me) ppm; ${ }^{13} \mathrm{C}$ NMR $\left(\mathrm{CDCl}_{3}\right): \delta$ 152.4, 146.8, 141.0, $140.9,138.9,138.5,138.0,137.7,132.1,130.8,129.4,128.3,128.2,126.8,126.4,116.2,112.0,56.7,21.4$, $21.2 \mathrm{ppm} ; m / z=688.2397$, calcd for $\left(\mathrm{C}_{43} \mathrm{H}_{38} \mathrm{~N}_{5} \mathrm{Zn}\right)^{+}=688.2413\left[(\mathrm{M}+\mathrm{H})^{+}\right]$.

\section{$N$-Benzyl-5,15-dimesityl-10-azacorrole 3a.}

A flask containing $N$-benzyl-5,15-dimesityl-10-azacorrole Zn(II) complex (7.10 mg, $10.3 \mu \mathrm{mol}$ ) was charged with $\mathrm{CHCl}_{3}(4.00 \mathrm{~mL})$. To the flask, trifluoroacetic acid $(1.00 \mathrm{~mL})$ was added. The mixture was stirred at r.t. for 10 minutes. The reaction was quenched with $\mathrm{NaHCO}_{3}$ aq. and extracted with $\mathrm{CHCl}_{3}$. The organic layer was washed with water, dried over anhydrous $\mathrm{Na}_{2} \mathrm{SO}_{4}$, and concentrated in vacuo. Purification by silica-gel column chromatography with $\mathrm{CHCl}_{3} /$ hexane afforded the title compound in $98 \%(6.30 \mathrm{mg}, 10.1 \mu \mathrm{mol})$ as a red solid. ${ }^{1} \mathrm{H}$ NMR (acetone- $\left.d_{6}\right): \delta 8.33\left(\mathrm{dd}, J^{1}=4.0 \mathrm{~Hz}, J^{2}=1.5 \mathrm{~Hz}, 2 \mathrm{H}\right.$, pyrrole- $\left.\beta\right), 8.15\left(\mathrm{dd}, J^{1}=4.8 \mathrm{~Hz}, J^{2}\right.$ $=0.75 \mathrm{~Hz}, 2 \mathrm{H}$, pyrrole- $\beta$ ), $7.98\left(\mathrm{~d}, J=4.5 \mathrm{~Hz}, 2 \mathrm{H}\right.$, pyrrole- $\beta$ ), $7.85\left(\mathrm{dd}, J^{1}=4.0 \mathrm{~Hz}, J^{2}=1.0 \mathrm{~Hz}, 2 \mathrm{H}\right.$, pyrrole- $\beta$ ), 7.26-7.31 (m, 5H, Ph), 7.21 (s, 4H, Mes), 7.12 (s, 2H, $\mathrm{CH}_{2}$ ), 5.23 (br s, 2H, NH), 2.51 (s, 6H, p-Me), 1.96 (s, 12H, o-Me) ppm; ${ }^{13} \mathrm{C}$ NMR $\left(\mathrm{CDCl}_{3}\right)$ : $\delta 147.0,138.6,138.5,138.1,137.7,137.1,136.6,134.9$, $130.2,129.2,128.5,128.0,127.9,125.5,125.4,116.0,112.2,57.3,21.5,21.1 \mathrm{ppm} ;$ HR-MS (ESI-MS): $\mathrm{m} / z=$ 626.3255, calcd for $\left(\mathrm{C}_{43} \mathrm{H}_{40} \mathrm{~N}_{5}\right)^{+}=626.3278\left[(\mathrm{M}+\mathrm{H})^{+}\right]$.

\section{$N$-Benzyl-5,15-diphenyl-10-azacorrole $3 b$.}

A Schlenk tube containing $N, N$-bis(meso-phenyl-1-bromodipyrrin-9-yl)- $N$-benzylamine $\mathrm{Zn}(\mathrm{II})$ complex (27.4 $\mathrm{mg}, 35.8 \mu \mathrm{mol})$, 2,2'-bipyridyl (14.8 mg, $94.8 \mu \mathrm{mol})$, and $\mathrm{Ni}(\mathrm{cod})_{2}(25.1 \mathrm{mg}, 91.3 \mu \mathrm{mol})$ was evacuated and then refilled with $\mathrm{N}_{2}$. To the tube, 1,5-cyclooctadiene $(9.00 \mu \mathrm{L}, 85.8 \mu \mathrm{mol})$ and dry DMF $(4.00 \mathrm{~mL})$ were added. The mixture was stirred at $50{ }^{\circ} \mathrm{C}$ for $1 \mathrm{~h}$. The reaction mixture was extracted with EtOAc. The organic layer was washed with water, dried over anhydrous $\mathrm{Na}_{2} \mathrm{SO}_{4}$, and concentrated in vacuo. Purification by silica-gel column chromatography with EtOAc/hexane afforded the title compound in 89\% (17.2 mg, 31.8 $\mu \mathrm{mol})$ as a red solid. ${ }^{1} \mathrm{H}$ NMR (acetone- $\left.d_{6}\right): \delta 8.45\left(\mathrm{dd}, J^{1}=4.0 \mathrm{~Hz}, J^{2}=1.5 \mathrm{~Hz}, 2 \mathrm{H}\right.$, pyrrole- $\left.\beta\right), 8.35(\mathrm{~d}, J=$ 
$4.0 \mathrm{~Hz}, 2 \mathrm{H}$, pyrrole- $\beta$ ), 8.29 (d, $J=4.5 \mathrm{~Hz}, 2 \mathrm{H}$, pyrrole- $\beta$ ), 8.09-8.14 (m, 6H), 7.73-7.79 (m, 6H, Ar- Ph), 7.26-7.28 (m, 5H, Bn-Ph), $7.21\left(\mathrm{~s}, 2 \mathrm{H}, \mathrm{CH}_{2}\right), 5.20$ (br s, $\left.2 \mathrm{H}, \mathrm{NH}\right) \mathrm{ppm} ;{ }^{13} \mathrm{C} \mathrm{NMR}\left(\mathrm{CDCl}_{3}\right): \delta 147.0,139.0$, $138.5,138.1,137.5,137.1,133.8,131.5,129.8,129.2,128.2,128.1,127.8,126.7,125.4,116.3,112.4,57.5$ ppm; HR-MS (ESI-MS): $m / z=542.2326$, calcd for $\left(\mathrm{C}_{37} \mathrm{H}_{28} \mathrm{~N}_{5}\right)^{+}=542.2339\left[(\mathrm{M}+\mathrm{H})^{+}\right]$.

\section{$N$-Benzyl-meso-3,5-dimethoxyphenyl-10-azacorrole 3c.}

A Schlenk tube containing $N, N$-Bis(meso-3,5-dimethoxyphenyl-1-bromodipyrrin-9-yl)- $N$-benzylamine $\mathrm{Zn}$ (II) complex (24.2 mg, $27.3 \mu \mathrm{mol}), 2,2$ '-bipyridyl (9.00 mg, $57.6 \mu \mathrm{mol})$, and $\mathrm{Ni}(\mathrm{cod})_{2}(17.6 \mathrm{mg}, 60.7 \mu \mathrm{mol})$ was evacuated and then refilled with $\mathrm{N}_{2}$. To the tube, 1,5-cyclooctadiene (7.00 $\left.\mu \mathrm{L}, 66.7 \mu \mathrm{mol}\right)$ and dry DMF (3.00 $\mathrm{mL}$ ) were added. The mixture was stirred at $50{ }^{\circ} \mathrm{C}$ for $1 \mathrm{~h}$. The reaction mixture was extracted with EtOAc. The organic layer was washed with water, dried over anhydrous $\mathrm{Na}_{2} \mathrm{SO}_{4}$, and concentrated in vacuo. Purification by silica-gel column chromatography with EtOAc/hexane afforded the title compound in $81 \%$ (14.6 mg, $22.1 \mu \mathrm{mol})$ as a red solid. ${ }^{1} \mathrm{H}$ NMR (acetone- $\left.d_{6}\right): \delta 8.45-8.47(\mathrm{~m}, 4 \mathrm{H}$, pyrrole- $\beta$ ), $8.28(\mathrm{~d}, J=4.5 \mathrm{~Hz}$, $2 \mathrm{H}$, pyrrole- $\beta$ ), $8.23\left(\mathrm{dd}, J^{1}=4.0 \mathrm{~Hz}, J^{2}=1.0 \mathrm{~Hz}, 2 \mathrm{H}\right.$, pyrrole- $\beta$ ), 7.24-7.31 (m, 9H), $7.21\left(\mathrm{~s}, 2 \mathrm{H}, \mathrm{CH}_{2}\right), 6.85$ (t, $J=2.3 \mathrm{~Hz}, 2 \mathrm{H}$, $5.13(\mathrm{br} \mathrm{s}, 2 \mathrm{H}, \mathrm{NH}) 3.98(\mathrm{~s}, 12 \mathrm{H}, \mathrm{OMe}) \mathrm{ppm} ;{ }^{13} \mathrm{C} \mathrm{NMR}\left(\mathrm{CDCl}_{3}\right): \delta 160.0,147.0,140.9$, 137.9, 137.6, 136.9, 131.4, 129.4, 129.2, 128.1, 126.7, 125.4, 116.3, 112.5, 112.4, 100.6, 55.8 ppm; HR-MS (ESI-MS): $m / z=662.2736$, calcd for $\left(\mathrm{C}_{41} \mathrm{H}_{36} \mathrm{~N}_{5} \mathrm{O}_{4}\right)^{+}=662.2762\left[(\mathrm{M}+\mathrm{H})^{+}\right]$.

\section{$N$-Benzyl-5,15-dimesityl-10-azacorrole $\mathrm{Cu}(\mathrm{II})$ complex 4a.}

A flask containing $N$-benzyl-5,15-dimesityl-10-azacorrole $(10.7 \mathrm{mg}, 17.1 \mu \mathrm{mol})$ and $\mathrm{Cu}(\mathrm{OAc})_{2} \bullet \mathrm{H}_{2} \mathrm{O}(35.4 \mathrm{mg}$, $177 \mu \mathrm{mol})$ was charged with $\mathrm{CHCl}_{3}(7.00 \mathrm{~mL})$ and $\mathrm{MeOH}(2.00 \mathrm{~mL})$. The mixture was stirred at $60{ }^{\circ} \mathrm{C}$ for 10 h. The reaction mixture was extracted with $\mathrm{CHCl}_{3}$. The organic layer was washed with water, dried over anhydrous $\mathrm{Na}_{2} \mathrm{SO}_{4}$, and concentrated in vacuo. Purification by silica-gel column chromatography with $\mathrm{CHCl}_{3} /$ hexane afforded the title compound in $92 \%(10.8 \mathrm{mg}, 15.7 \mu \mathrm{mol})$ as a purple solid. HR-MS (ESI-MS): $m / z=687.2403$, calcd for $\left(\mathrm{C}_{43} \mathrm{H}_{38} \mathrm{~N}_{5} \mathrm{Cu}\right)^{+}=687.2418\left[(\mathrm{M}+\mathrm{H})^{+}\right]$.

\section{$N$-benzyl-5,15-dimesityl-10-azacorrole Pd(II) complex $5 a$.}

A flask containing $N$-benzyl-5,15-dimesityl-10-azacorrole $(5.61 \mathrm{mg}, 8.96 \mu \mathrm{mol})$ and $\mathrm{Pd}(\mathrm{OAc})_{2}(11.4 \mathrm{mg}, 50.8$ $\mu \mathrm{mol})$ was charged with $\mathrm{CHCl}_{3}(5.00 \mathrm{~mL})$ and $\mathrm{MeOH}(2.00 \mathrm{~mL})$. The mixture was stirred at $60{ }^{\circ} \mathrm{C}$ for $3 \mathrm{~h}$. The reaction mixture was extracted with $\mathrm{CHCl}_{3}$. The organic layer was washed with water, dried over 
anhydrous $\mathrm{Na}_{2} \mathrm{SO}_{4}$, and concentrated in vacuo. Purification by silica-gel column chromatography with $\mathrm{CHCl}_{3} /$ hexane afforded the title compound in $63 \%(4.11 \mathrm{mg}, 5.63 \mu \mathrm{mol})$ as a black solid. ${ }^{1} \mathrm{H} \mathrm{NMR}\left(\mathrm{CDCl}_{3}\right): \delta$ $8.20(\mathrm{~d}, J=4.5 \mathrm{~Hz}, 2 \mathrm{H}$, pyrrole- $\beta$ ), 8.05 (d, $J=4.5 \mathrm{~Hz}, 2 \mathrm{H}$, pyrrole- $\beta$ ), 7.89 (d, $J=4.5 \mathrm{~Hz}, 2 \mathrm{H}$, pyrrole- $\beta$ ), 7.77 (d, $J=4.0 \mathrm{~Hz}, 2 \mathrm{H}$, pyrrole- $\beta$ ), 7.27-7.29 (m, 3H, Ph), 7.19-7.21 (m, 2H, Ph), 7.14 (s, 4H, Mes), 6.97 (s, $\left.2 \mathrm{H}, \mathrm{CH}_{2}\right), 2.53$ (s, 6H, p-Me), 1.95 (s, 12H,o-Me) ppm; ${ }^{13} \mathrm{C} \mathrm{NMR}\left(\mathrm{CDCl}_{3}\right): \delta$ 144.3, 142.7, 138.6, 138.3, 137.6, 135.6, 132.8, 132.2, 131.8, 131.4, 129.2, 128.2, 127.8, 125.6, 123.8, 118.3, 111.7, 57.1, 21.5, 21.2 ppm; HR-MS (ESI-MS): $m / z=730.2160$, calcd for $\left(\mathrm{C}_{43} \mathrm{H}_{38} \mathrm{~N}_{5} \mathrm{Pd}\right)^{+}=730.2172\left[(\mathrm{M}+\mathrm{H})^{+}\right]$. 


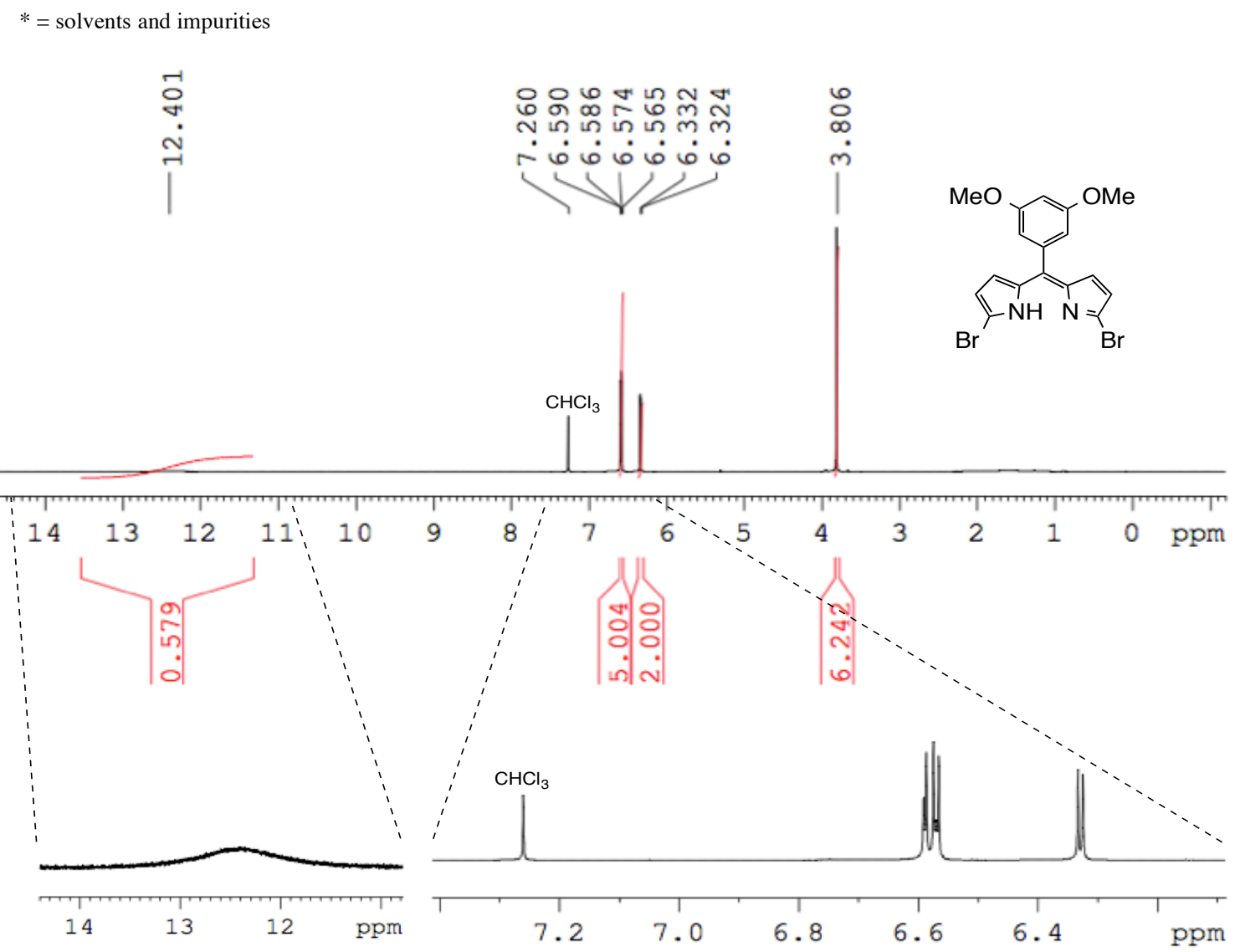

Figure S1. ${ }^{1} \mathrm{H}$ NMR spectrum of meso-3,5-dimethoxyphenyl- $\alpha, \alpha^{\prime}$-dibromodipyrrin in $\mathrm{CDCl}_{3}$.

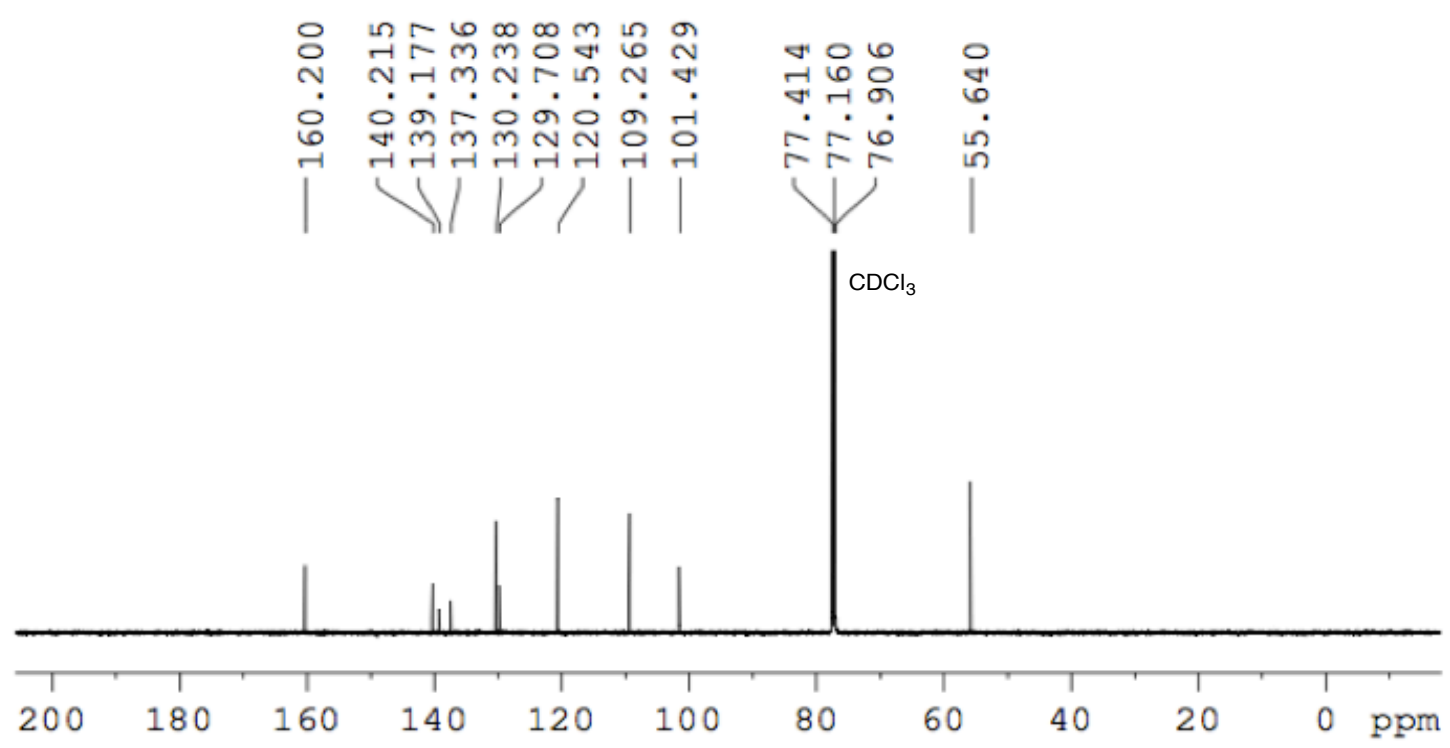

Figure S2. ${ }^{13} \mathrm{C}$ NMR spectrum of meso-3,5-dimethoxyphenyl- $\alpha, \alpha^{\prime}$-dibromodipyrrin in $\mathrm{CDCl}_{3}$. 


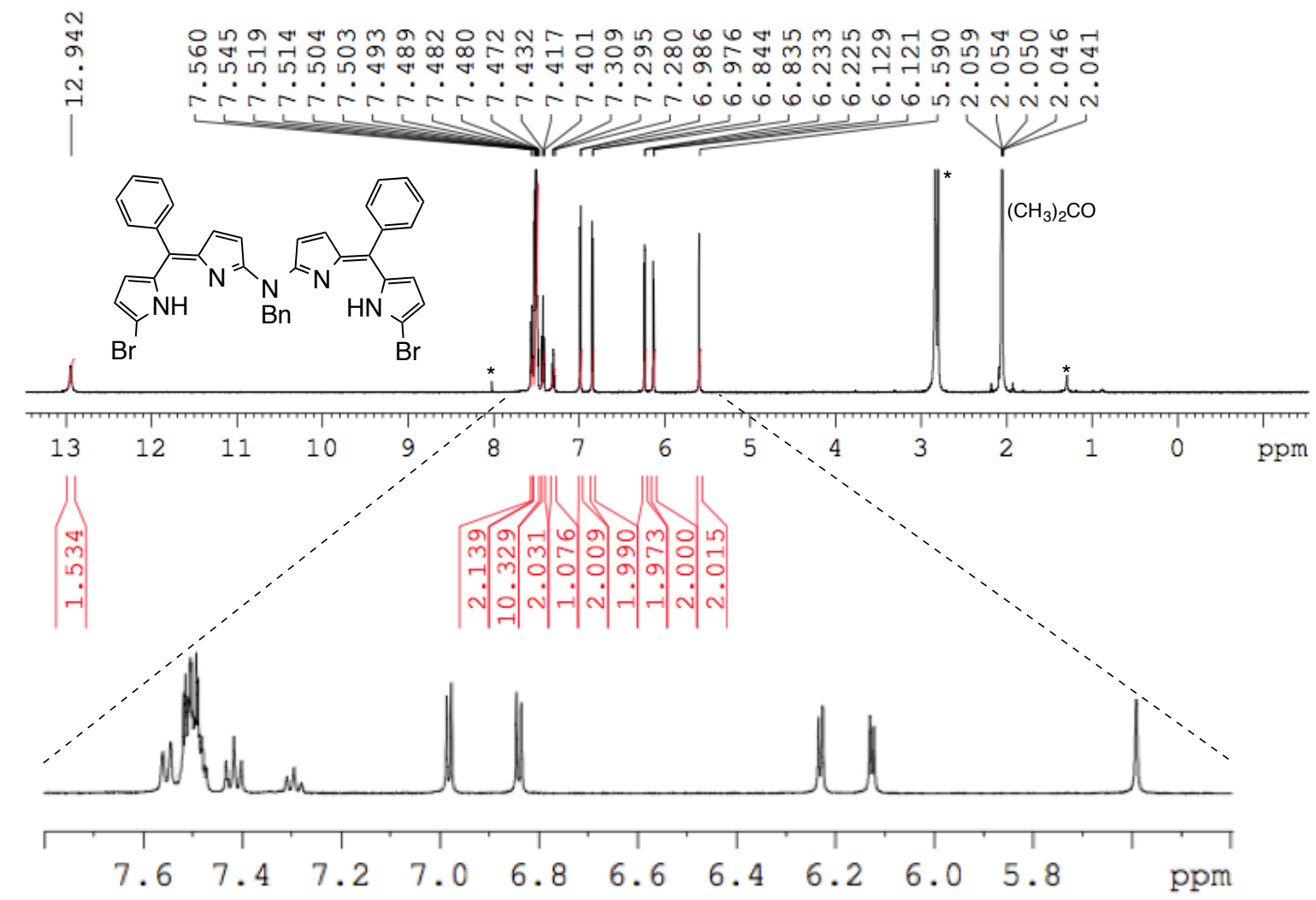

Figure S3. ${ }^{1} \mathrm{H}$ NMR spectrum of $N, N$-bis(meso-phenyl-1-bromodipyrrin-9-yl)- $N$-benzylamine in acetone- $d_{6}$.

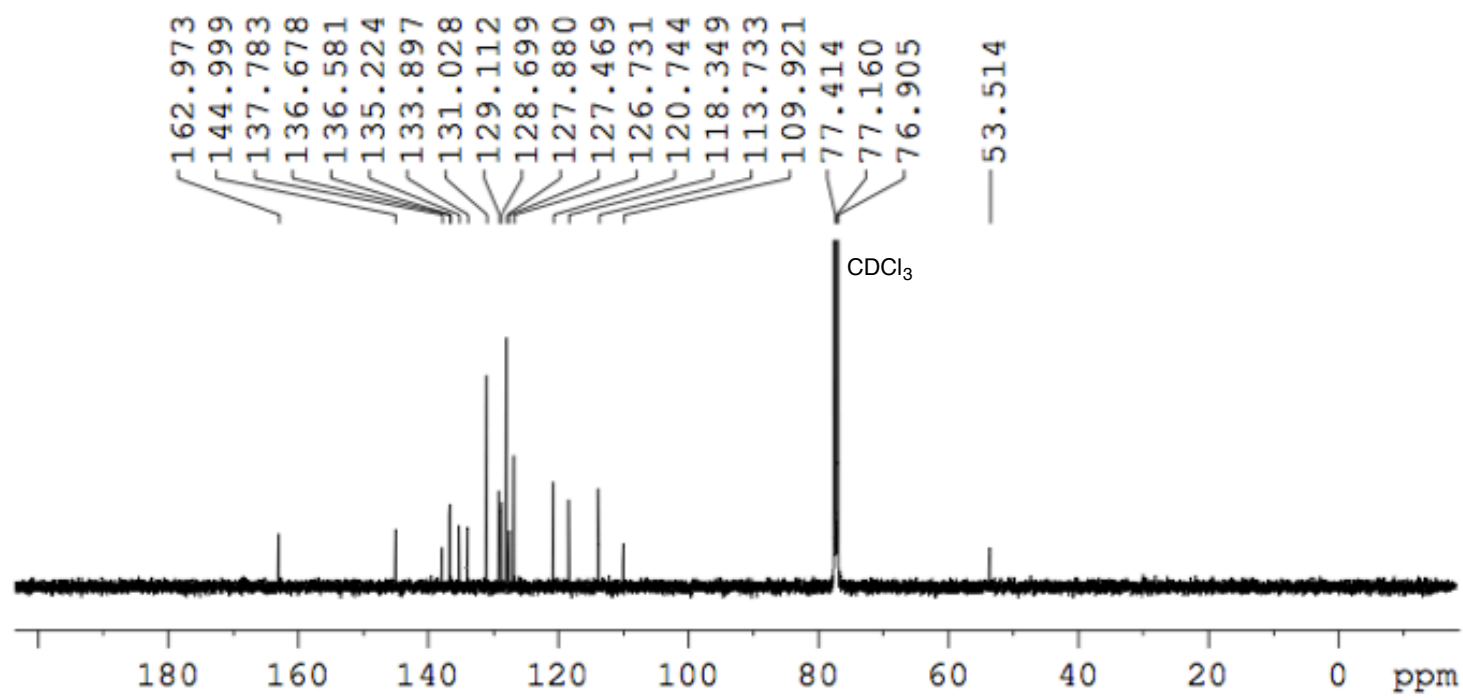

Figure S4. ${ }^{13} \mathrm{C}$ NMR spectrum of $N, N$-bis(meso-phenyl-1-bromodipyrrin-9-yl)- $N$-benzylamine in $\mathrm{CDCl}_{3}$. 


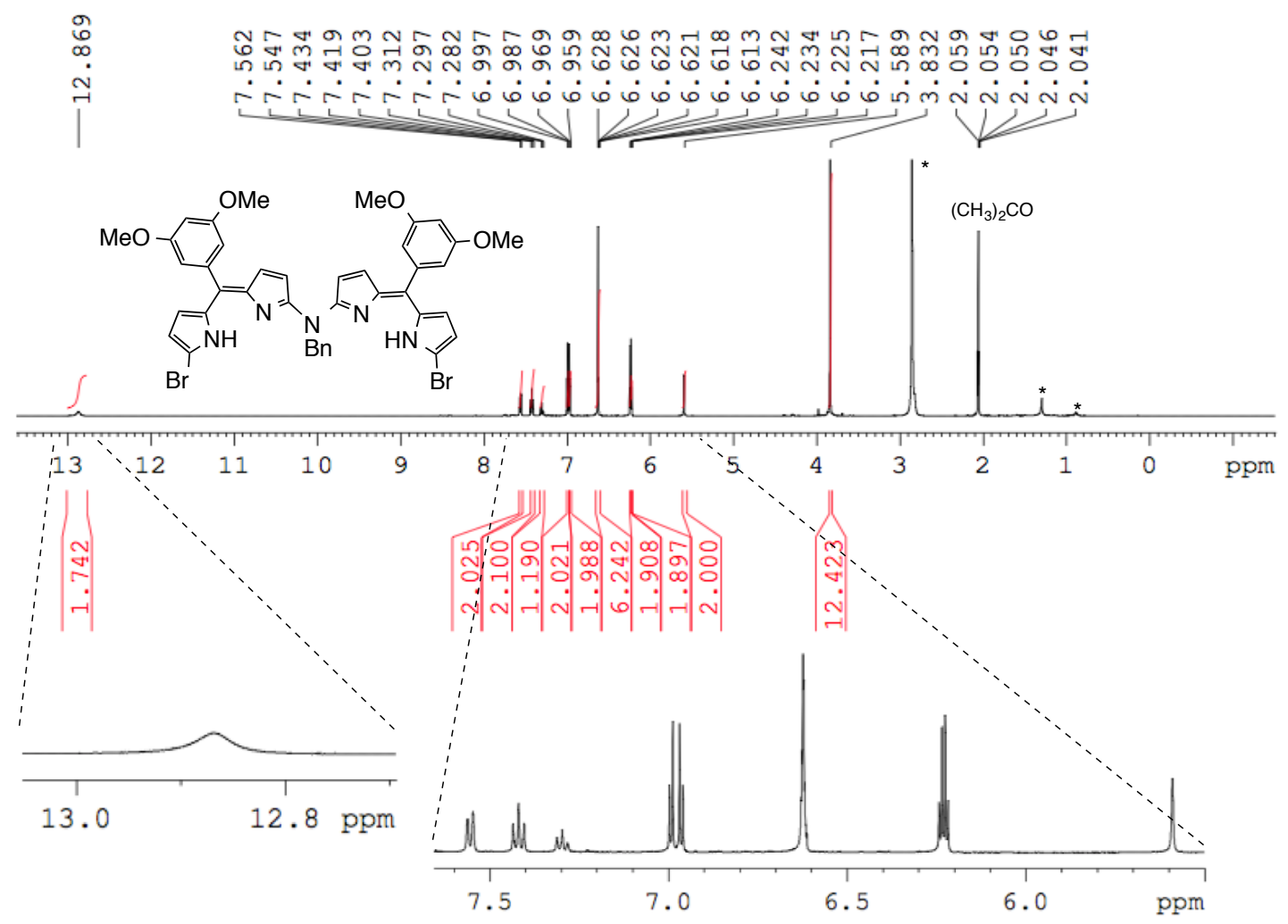

Figure S5. ${ }^{1} \mathrm{H}$ NMR spectrum of $N, N$-bis(meso-3,5-dimethoxyphenyl-1-bromodipyrrin-9-yl)- $N$-benzylamine in acetone- $d_{6}$.

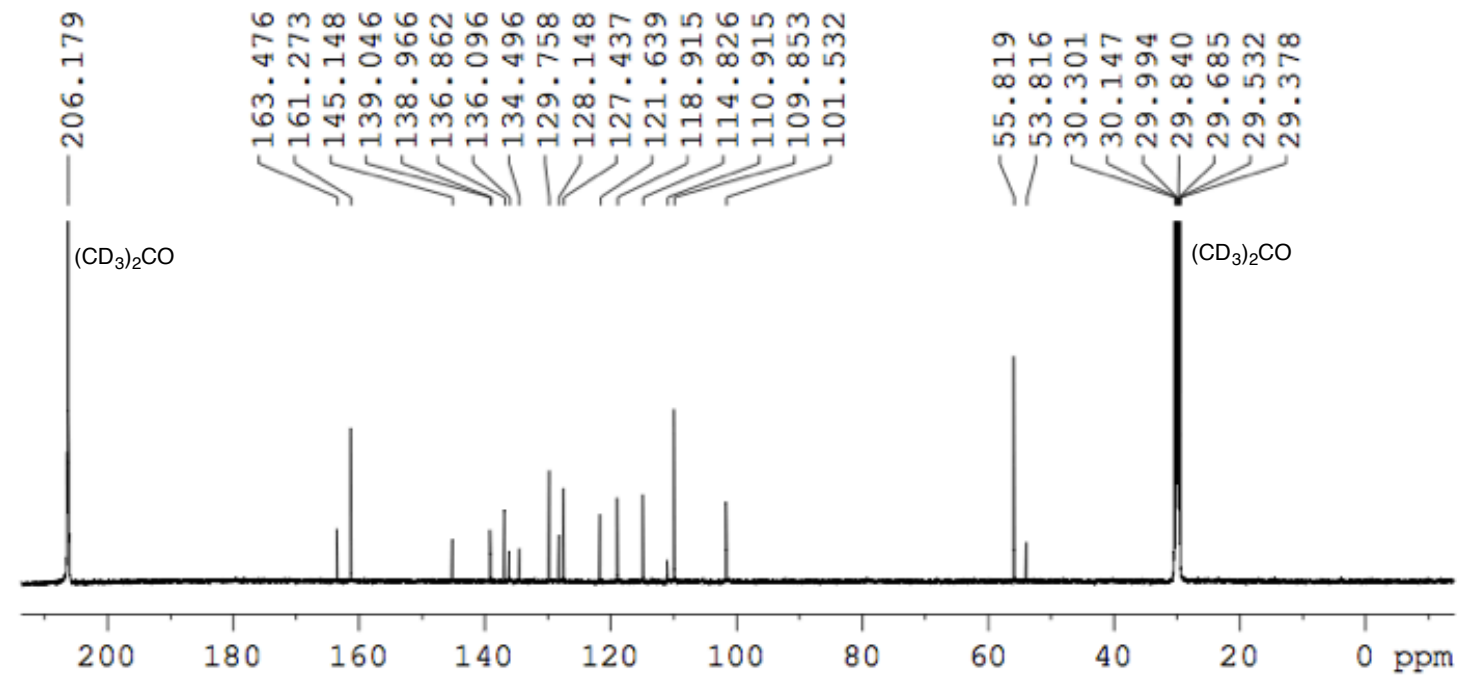

Figure S6. ${ }^{13} \mathrm{C}$ NMR spectrum of $N, N$-bis(meso-3,5-dimethoxyphenyl-1-bromodipyrrin-9-yl)- $N$-benzylamine in acetone- $d_{6}$. 


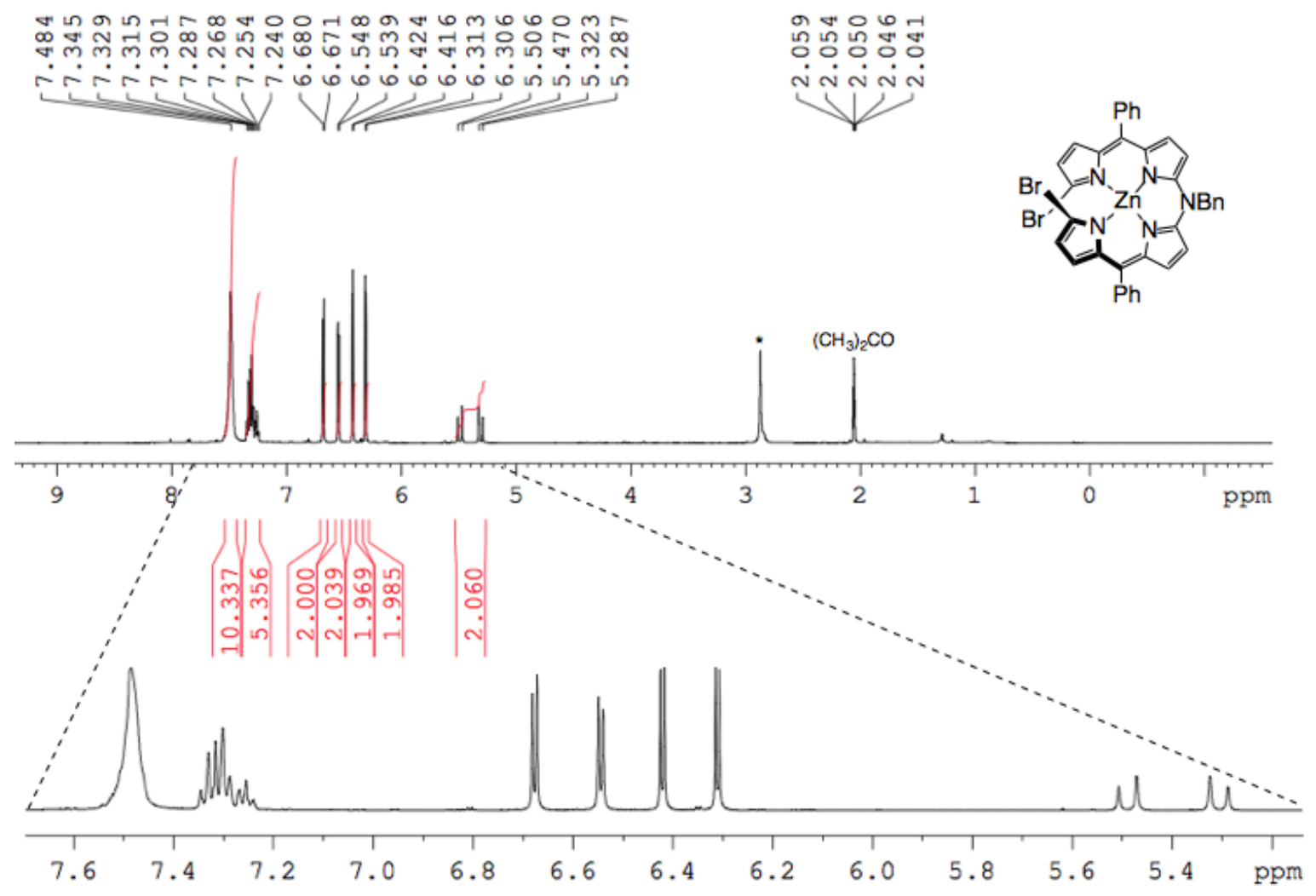

Figure S7. ${ }^{1} \mathrm{H}$ NMR spectrum of $\mathbf{1 b}$ in acetone- $d_{6}$.

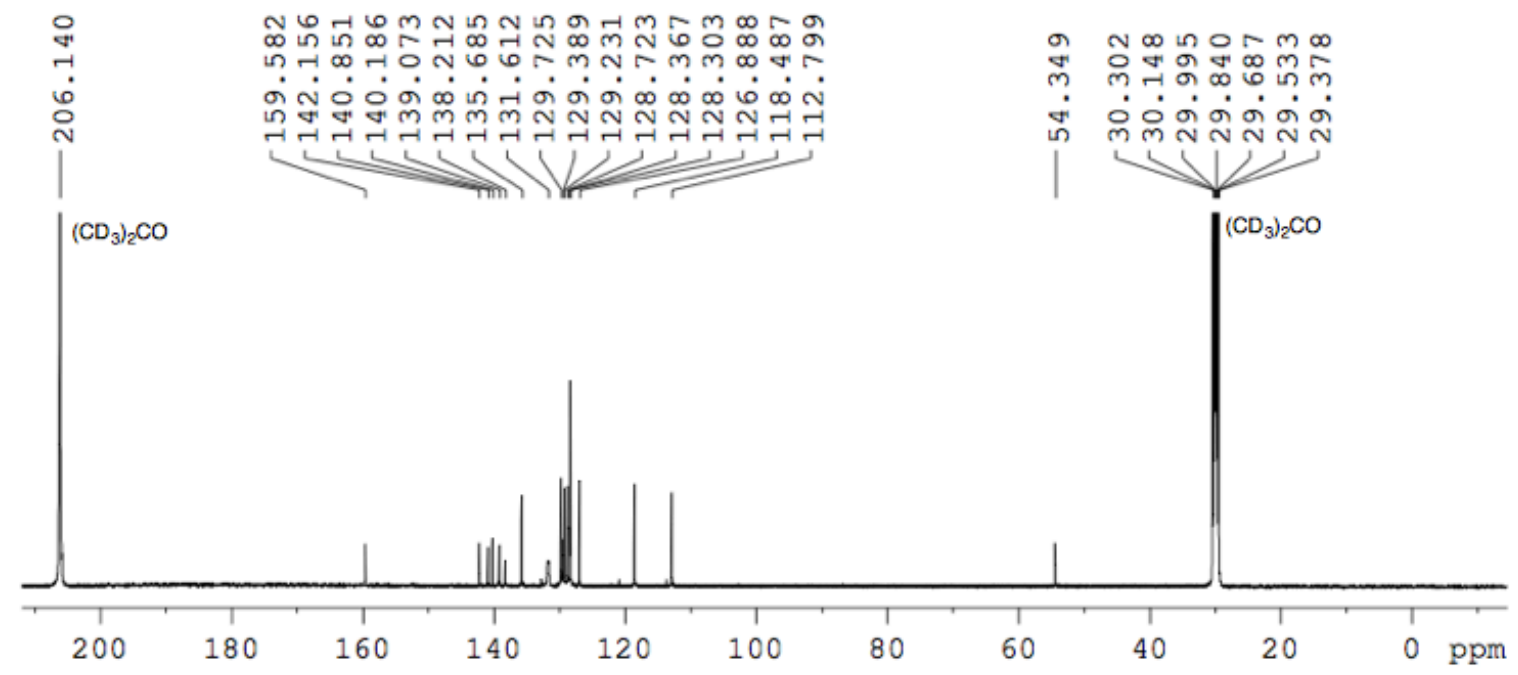

Figure S8. ${ }^{13} \mathrm{C}$ NMR spectrum of $\mathbf{1 b}$ in acetone- $d_{6}$. 


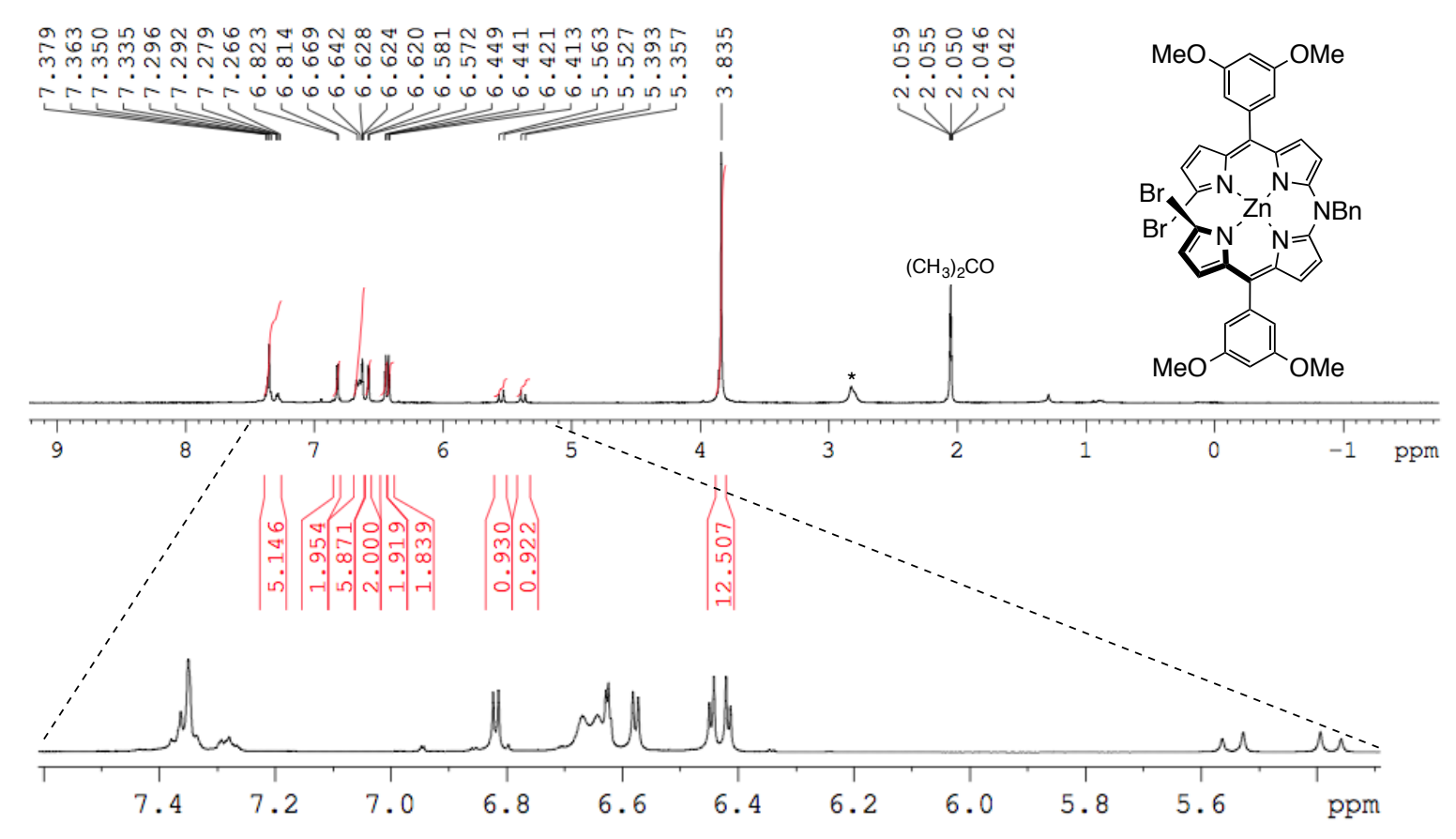

Figure S9. ${ }^{1} \mathrm{H}$ NMR spectrum of $1 \mathrm{c}$ in acetone- $d_{6}$.

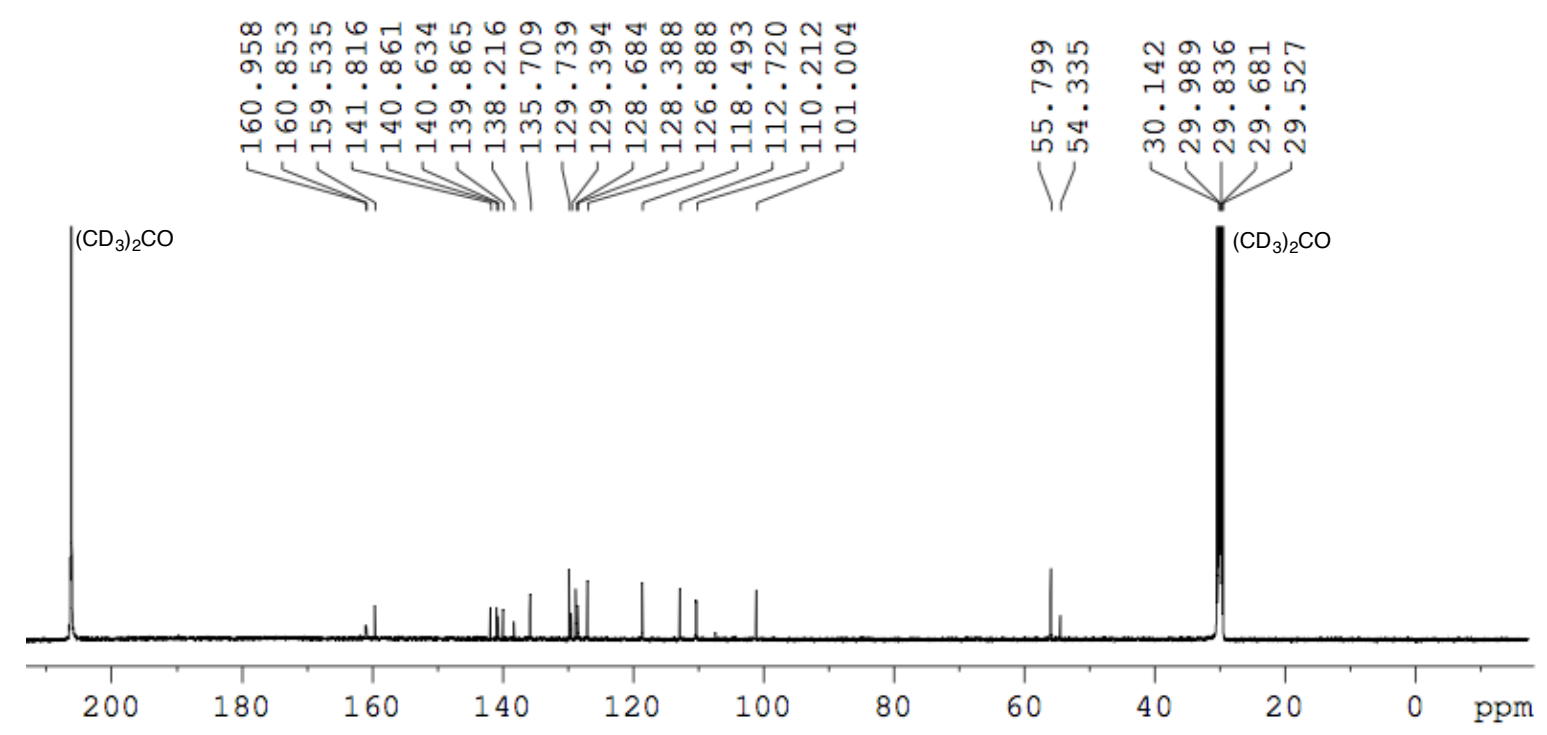

Figure S10. ${ }^{13} \mathrm{C}$ NMR spectrum of $1 \mathrm{c}$ in acetone- $d_{6}$. 


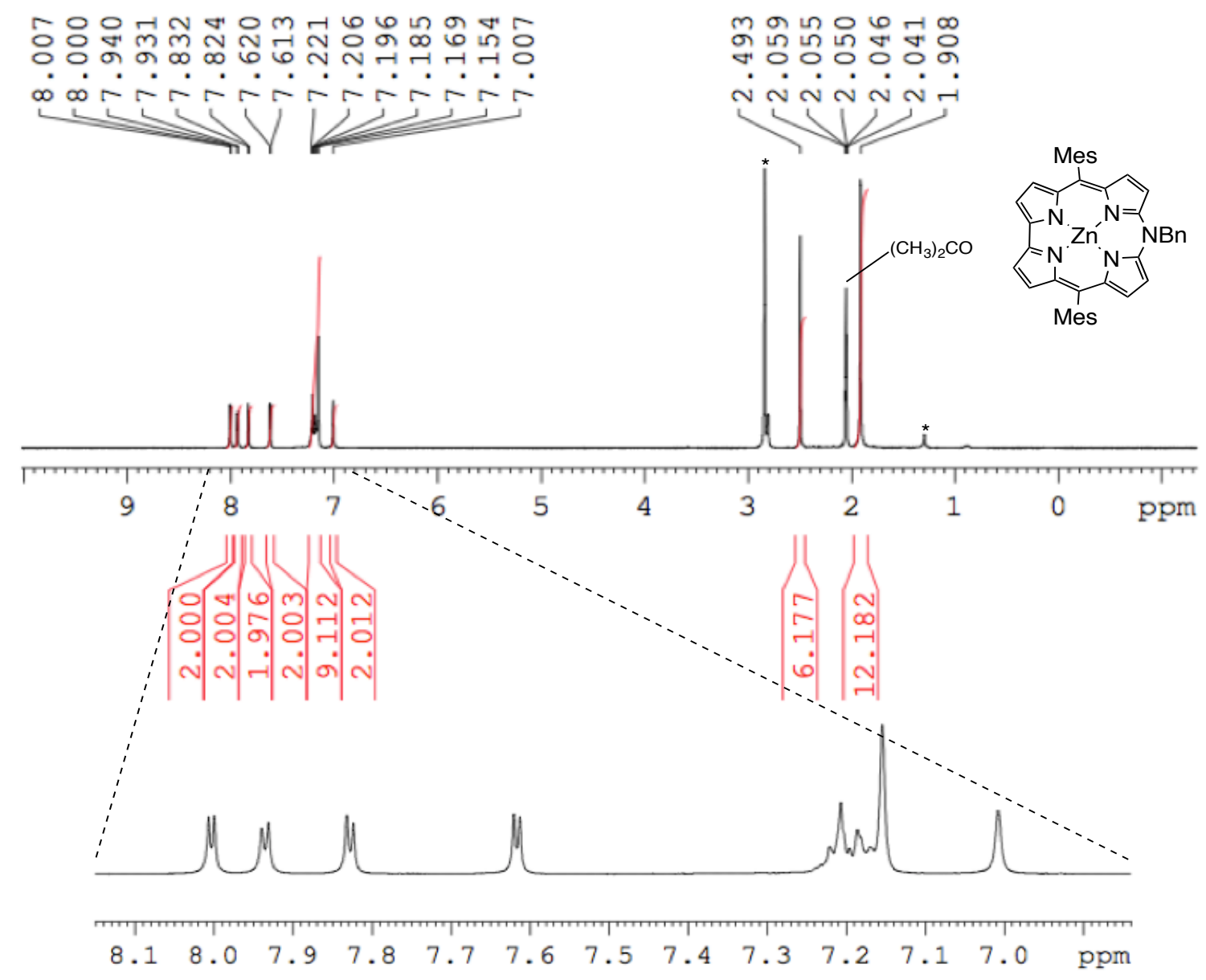

Figure S11. ${ }^{1} \mathrm{H}$ NMR spectrum of 2a in acetone- $d_{6}$.

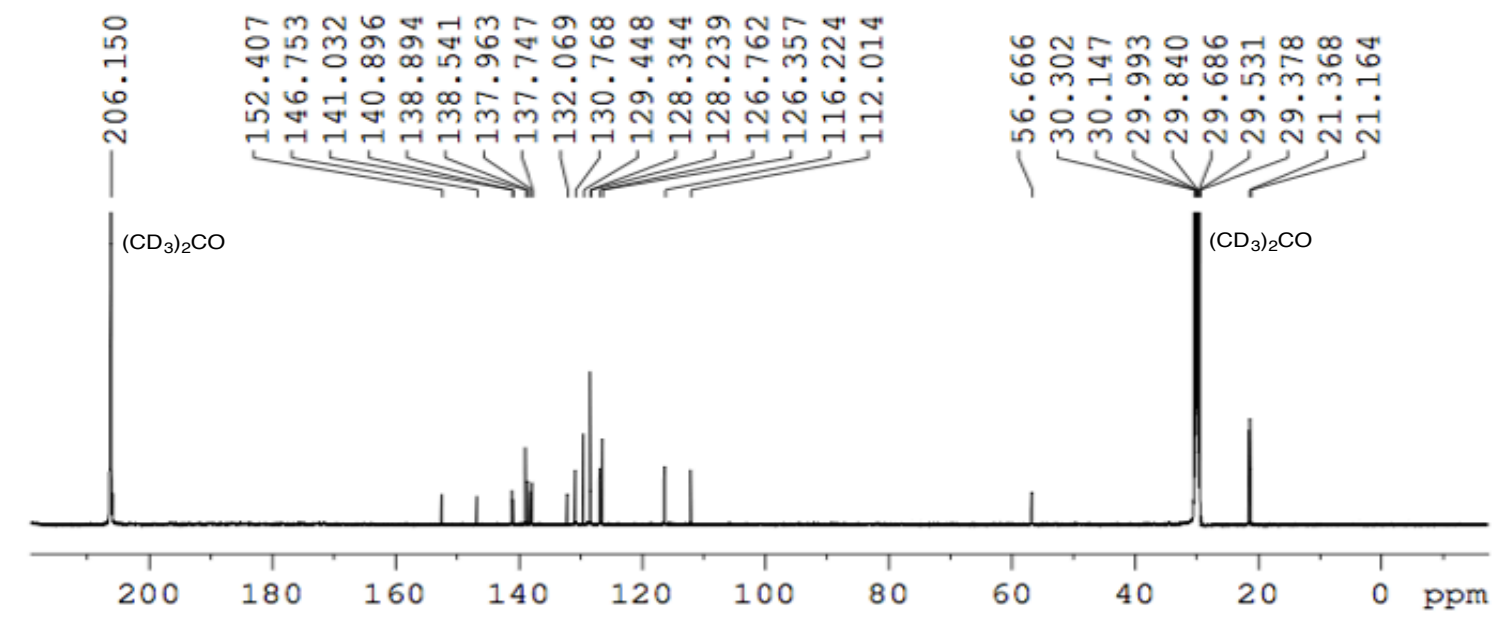

Figure $\mathbf{S 1 2} .{ }^{13} \mathrm{C}$ NMR spectrum of $2 \mathbf{a}$ in acetone- $d_{6}$. 


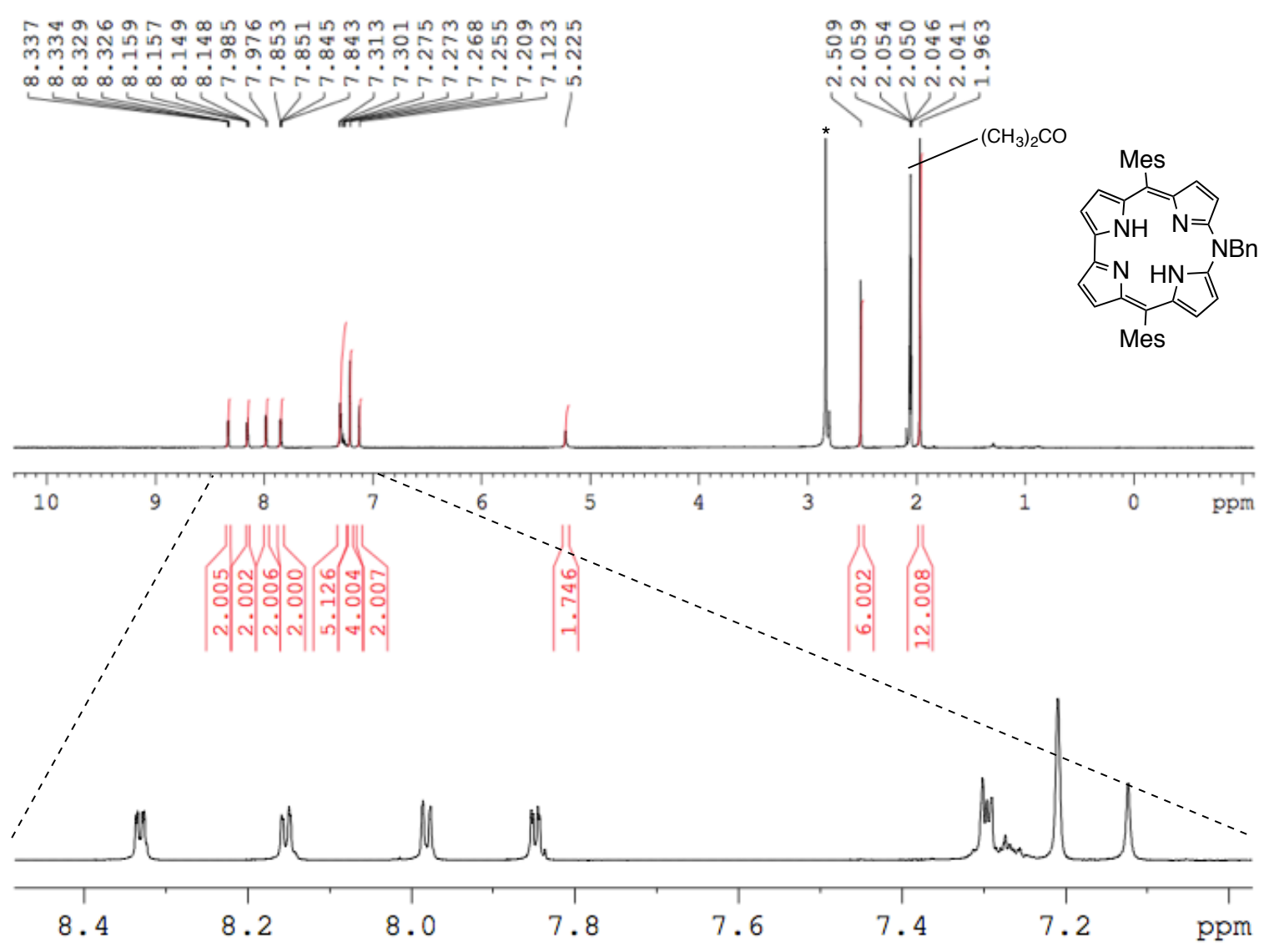

Figure S13. ${ }^{1} \mathrm{H}$ NMR spectrum of $\mathbf{3 a}$ in acetone- $d_{6}$.

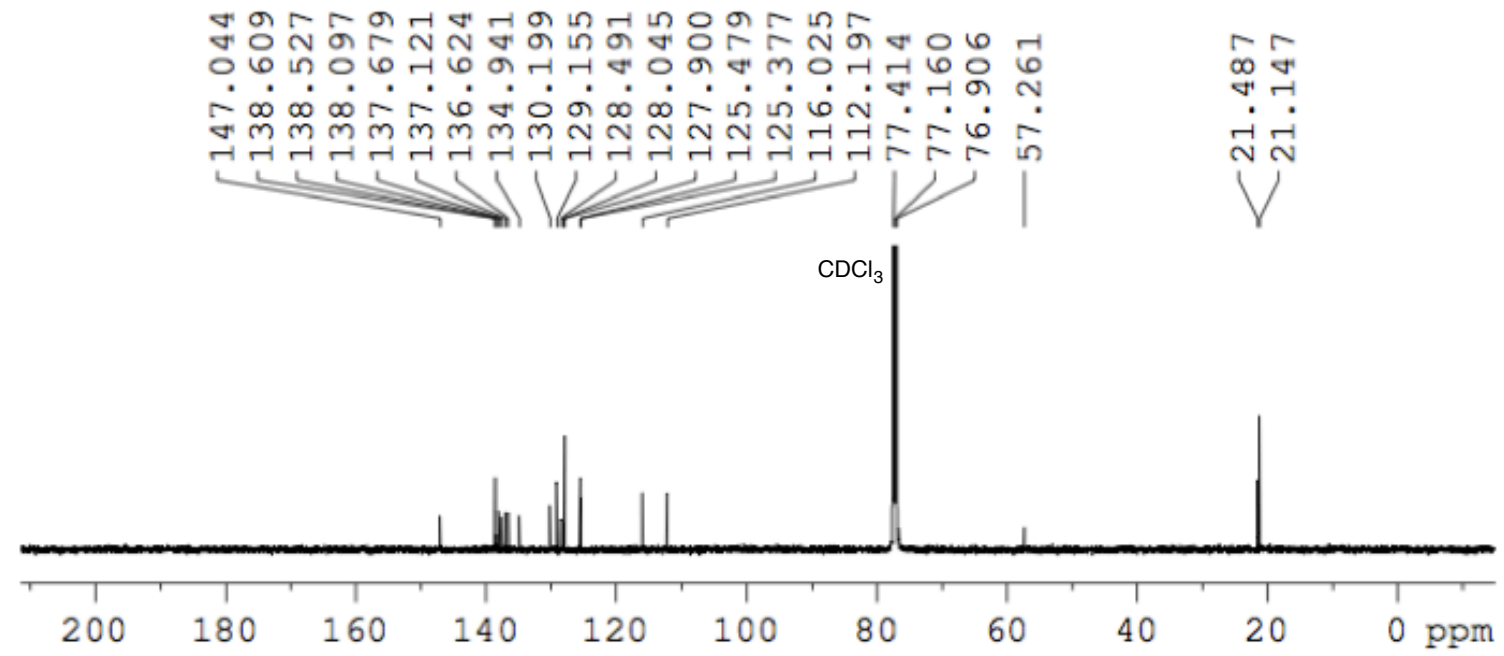

Figure S14. ${ }^{13} \mathrm{C}$ NMR spectrum of $3 a$ in $\mathrm{CDCl}_{3}$. 
은 A

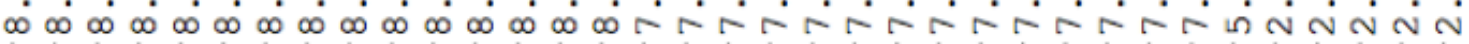

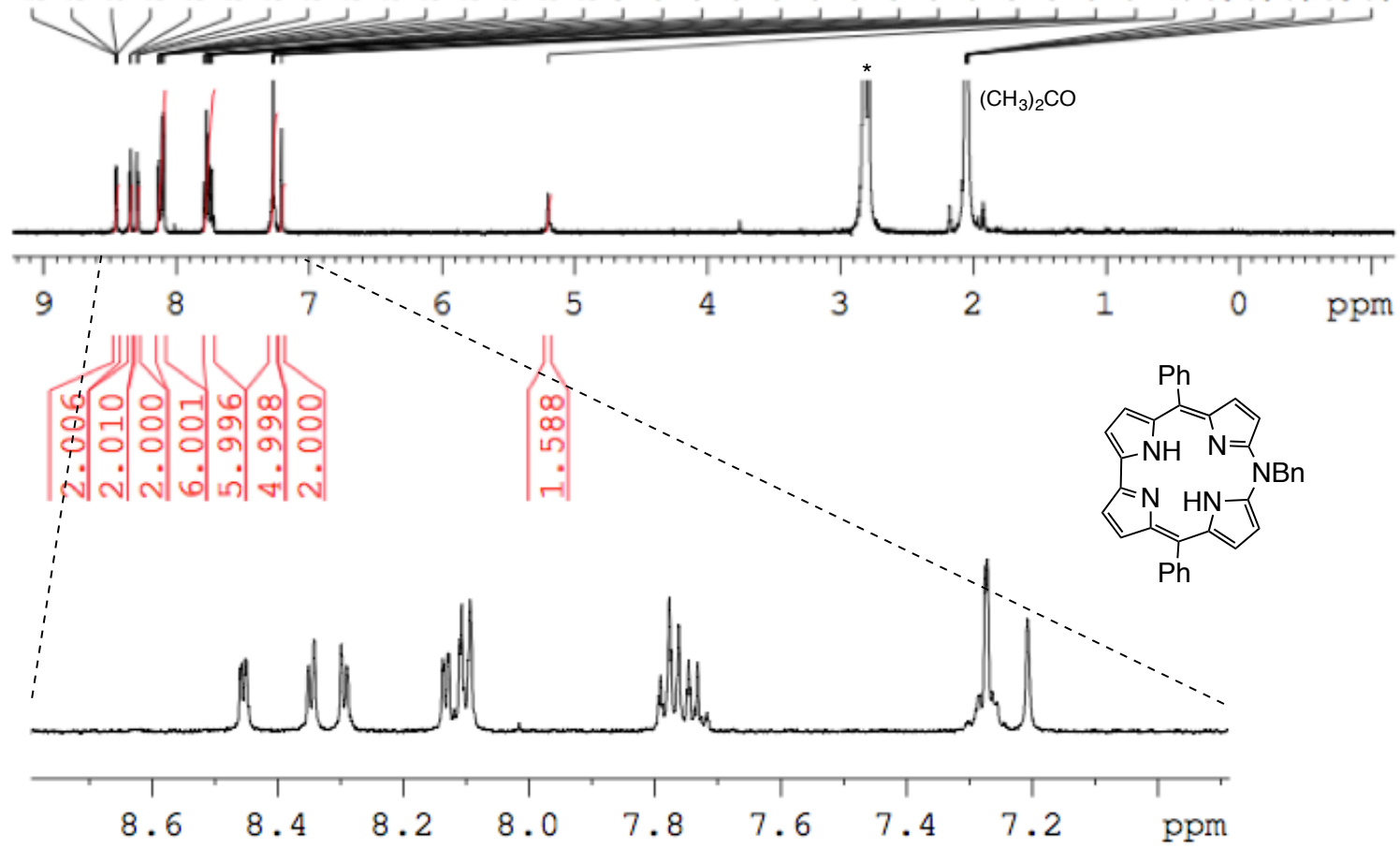

Figure S15. ${ }^{1} \mathrm{H}$ NMR spectrum of $\mathbf{3 b}$ in acetone- $d_{6}$.

๓

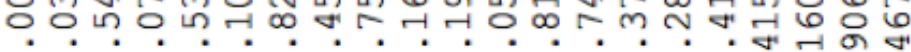

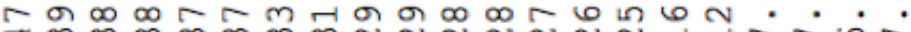
ำ mm mm $m$ m

$\mathrm{CDCl}_{3}$

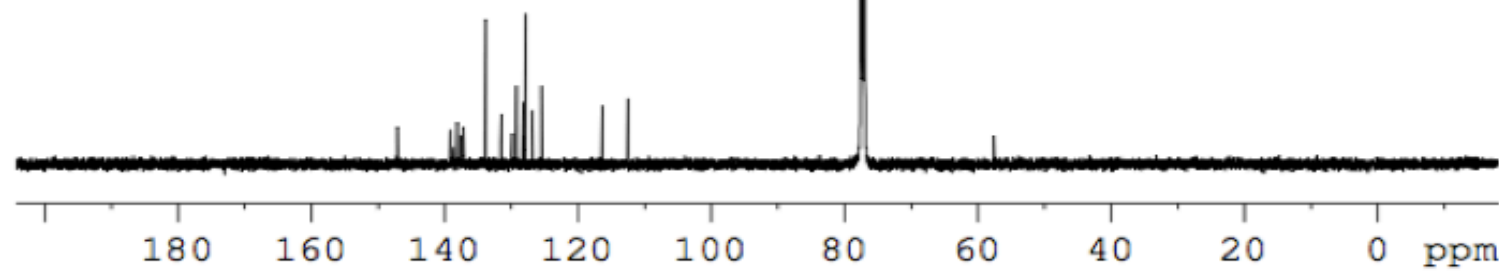

Figure S16. ${ }^{13} \mathrm{C}$ NMR spectrum of $\mathbf{3 b}$ in $\mathrm{CDCl}_{3}$. 


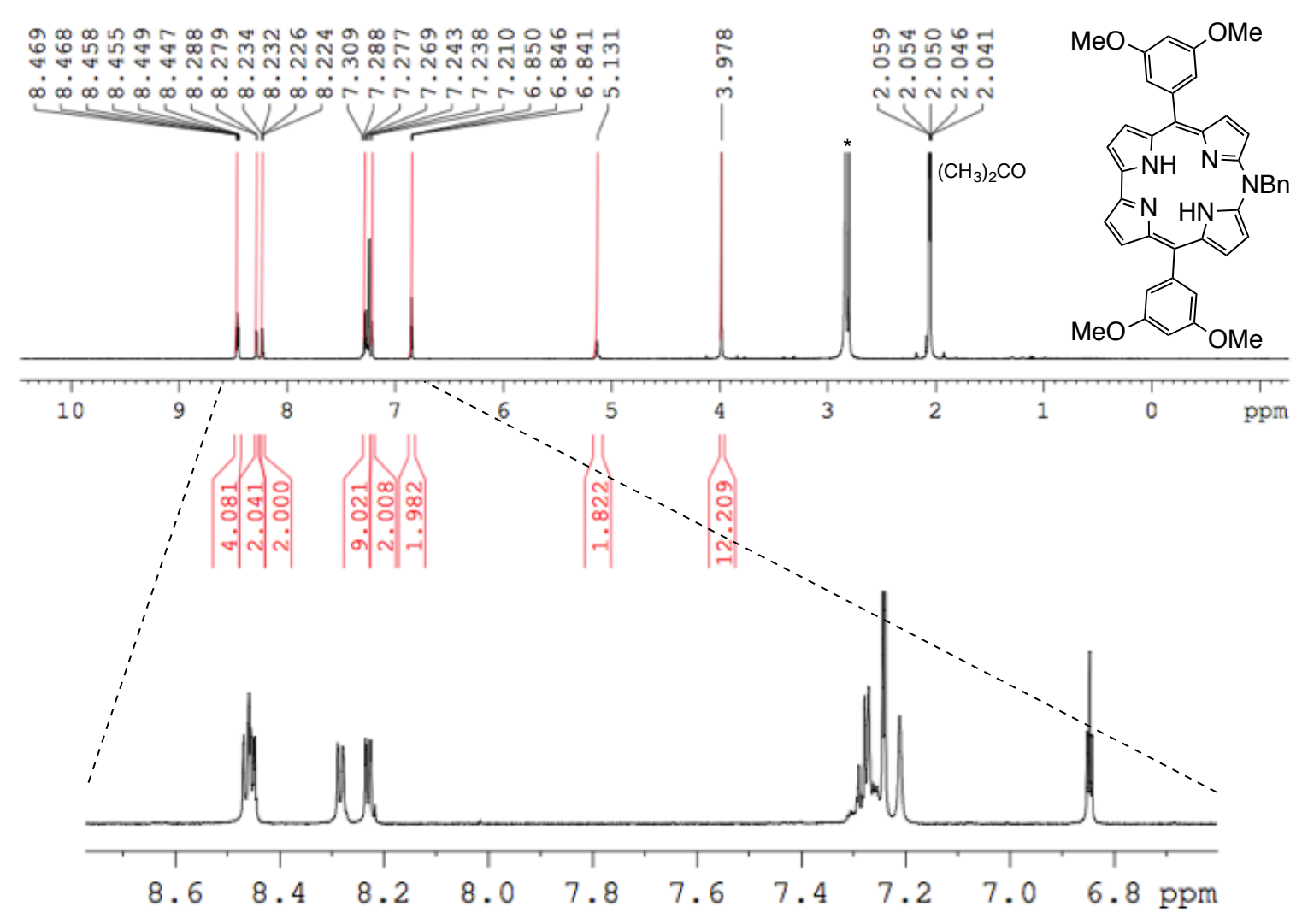

Figure S17. ${ }^{1} \mathrm{H}$ NMR spectrum of $\mathbf{3 c}$ in acetone- $d_{6}$.

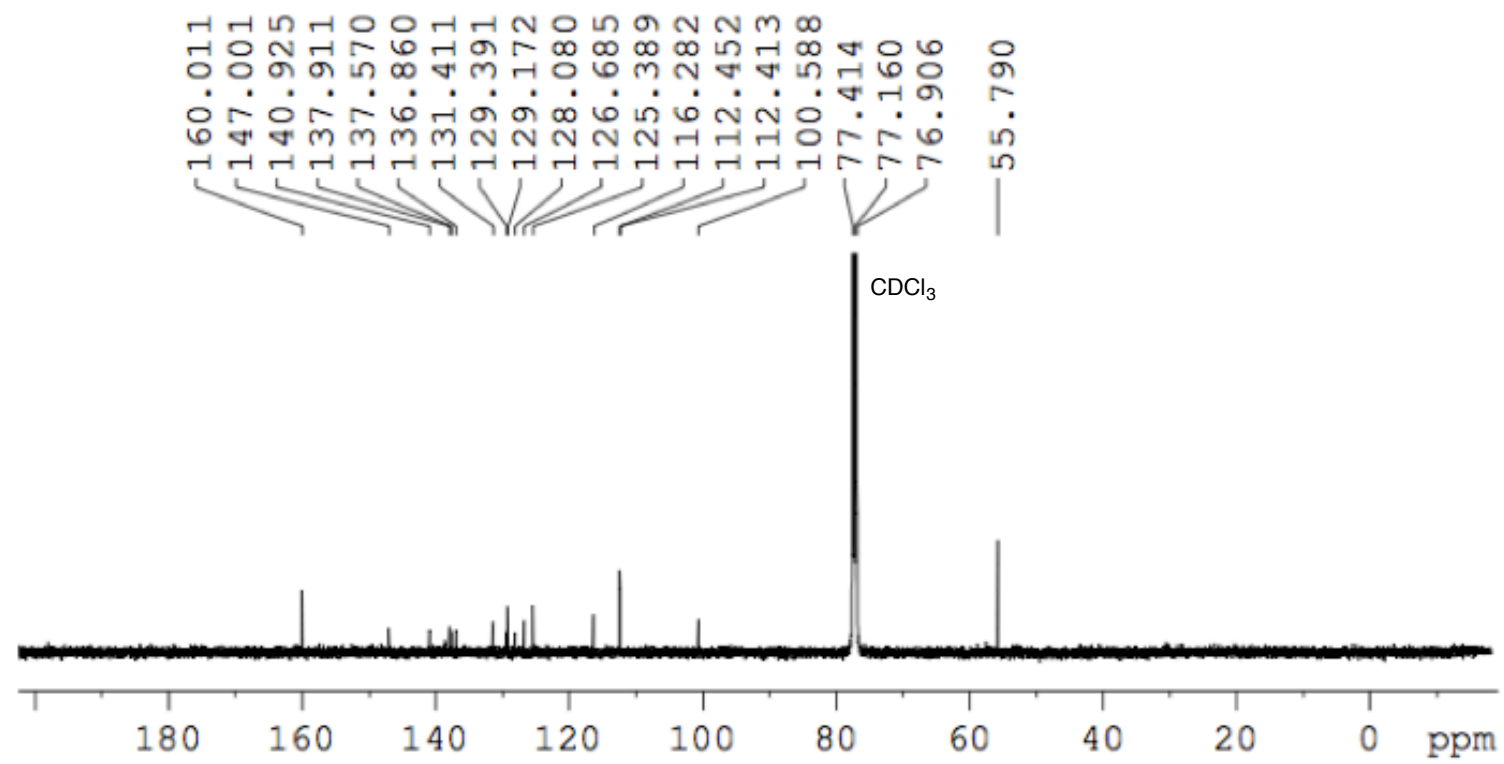

Figure S18. ${ }^{13} \mathrm{C}$ NMR spectrum of $3 \mathrm{c}$ in $\mathrm{CDCl}_{3}$. 


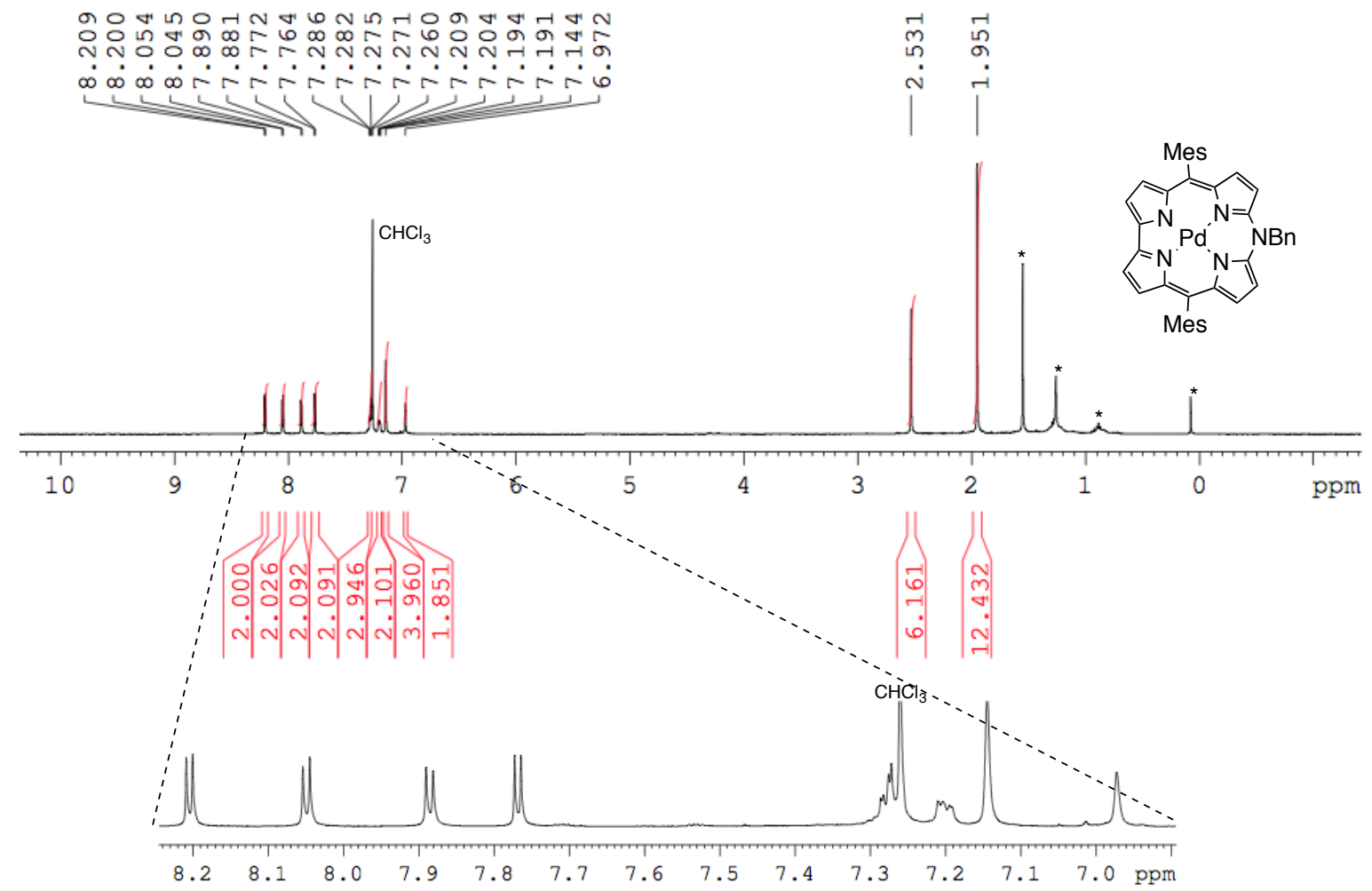

Figure S19. ${ }^{1} \mathrm{H}$ NMR spectrum of $\mathbf{5 a}$ in $\mathrm{CDCl}_{3}$.

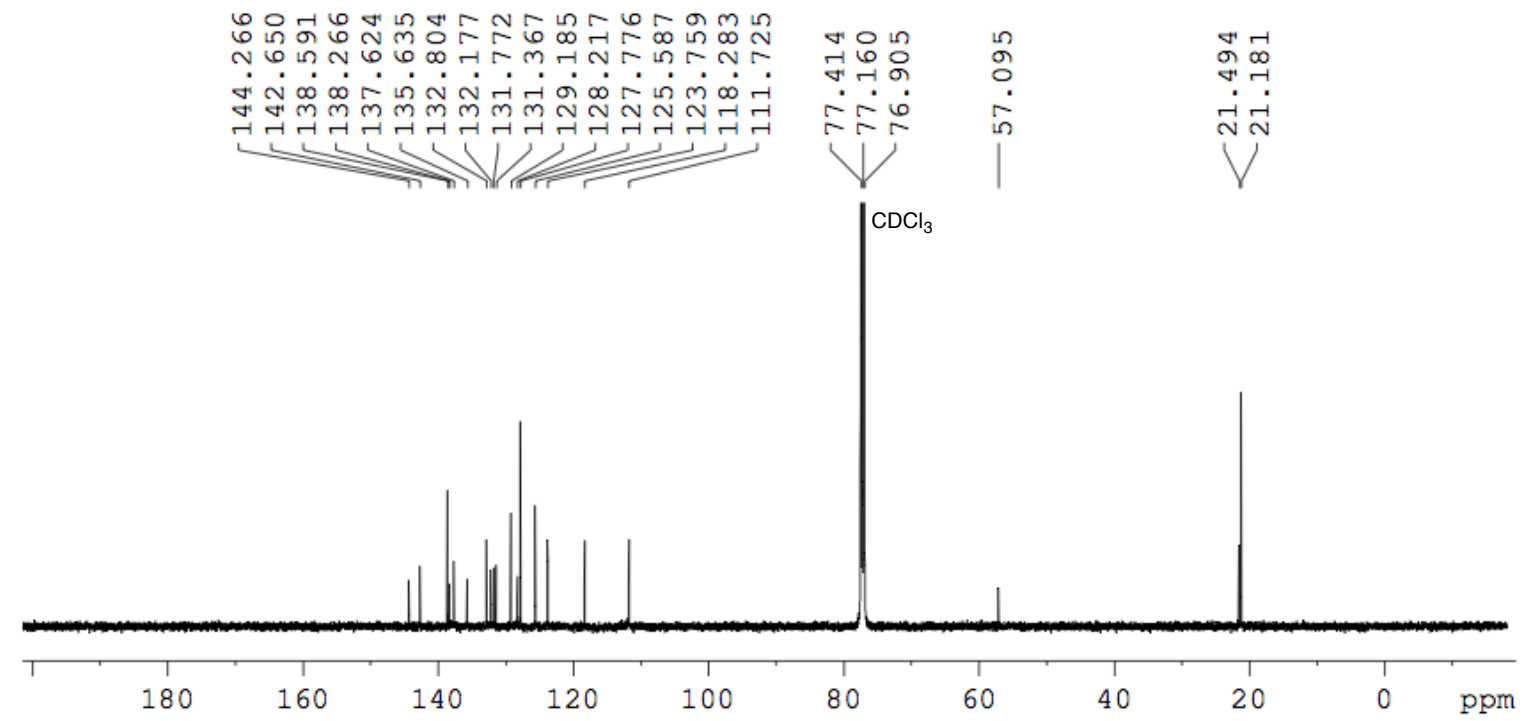

Figure S20. ${ }^{13} \mathrm{C}$ NMR spectrum of $\mathbf{5 a}$ in $\mathrm{CDCl}_{3}$. 
Table 1. Summary of X-ray crystallographic data of 2a, 3a, and 5a.

\begin{tabular}{|c|c|c|c|}
\hline & $2 a$ & $3 a$ & $5 a$ \\
\hline empirical formula & $\mathrm{C}_{45} \mathrm{H}_{45} \mathrm{~N}_{5} \mathrm{O}_{2} \mathrm{Zn}$ & $\mathrm{C}_{44} \mathrm{H}_{40} \mathrm{~N}_{5} \mathrm{Cl}_{2}$ & $\mathrm{C}_{45} \mathrm{H}_{41} \mathrm{~N}_{5} \mathrm{Br}_{1} \mathrm{Cl}_{2} \mathrm{Pd}$ \\
\hline moiety formula & $\mathrm{C}_{44} \mathrm{H}_{41} \mathrm{~N}_{5} \mathrm{OZn}, \mathrm{CH}_{4} \mathrm{O}$ & $\mathrm{C}_{43} \mathrm{H}_{39} \mathrm{~N}_{5}, 1 / 2\left(\mathrm{CHCl}_{3}\right)$ & $\mathrm{C}_{45} \mathrm{H}_{41} \mathrm{~N}_{5} \mathrm{Br}_{1} \mathrm{Cl}_{2} \mathrm{Pd}$ \\
\hline formula weight & 753.23 & 685.47 & 841.86 \\
\hline habit & prism & prism & prism \\
\hline$T, \mathrm{~K}$ & $93(2)$ & $93(2)$ & $93(2)$ \\
\hline crystal system & monoclinic & monoclinic & monoclinic \\
\hline space group & $P 2_{1} / C(14)$ & $P 2_{1} / C(14)$ & $P 2_{1} / c(14)$ \\
\hline$a, \AA$ & $19.5138(6)$ & $11.8403(11)$ & $16.4227(16)$ \\
\hline$b, \AA$ & $7.8297(2)$ & $23.720(2)$ & $20.557(2)$ \\
\hline$c, \AA$ & $25.5113(8)$ & $13.7832(13)$ & $12.5961(12)$ \\
\hline$\alpha$, deg & 90 & 90 & 90 \\
\hline$\beta$, deg & $105.1814(15)$ & $112.172(2)$ & $112.282(2)$ \\
\hline$\gamma$, deg & 90 & 90 & 90 \\
\hline$V, \AA^{3}$ & $3761.77(19)$ & $3584.8(6)$ & $3934.0(7)$ \\
\hline$z$ & 4 & 4 & 4 \\
\hline$D c, \mathrm{~g} / \mathrm{cm}^{3}$ & 1.330 & 1.270 & 1.421 \\
\hline$F(000)$ & 1584 & 1444 & 1726 \\
\hline crystal size, $\mathrm{mm}^{3}$ & $0.43 \times 0.12 \times 0.12$ & $0.26 \times 0.20 \times 0.082$ & $0.49 \times 0.43 \times 0.06$ \\
\hline $2 \theta_{\max }, \circ$ & 50.0 & 50.0 & 50.3 \\
\hline$R_{1}(I>2 \sigma(I))$ & 0.0481 & 0.0744 & 0.0739 \\
\hline$w R_{2}$ (all data) & 0.1288 & 0.2019 & 0.1829 \\
\hline GOF & 1.038 & 1.036 & 1.130 \\
\hline obs reflects & 5641 & 9000 & 5574 \\
\hline total reflects & 6606 & 11186 & 6588 \\
\hline parameters & 498 & 913 & 514 \\
\hline
\end{tabular}




\section{Electrochemical analysis}

The cyclic voltammogram and differential-pulse voltammogram of $\mathbf{3 a}, \mathbf{3 b}, \mathbf{3 c}, \mathbf{2 a}$, and $\mathbf{5 a}$ were recorded using an ALS electrochemical analyzer 612C. Measurements were performed in freshly distilled THF with tetrabutylammonium hexafluorophosphate as the electrolyte. A three-electrode system was used. The system consisted of a platinum working electrode, a platinum wire, and $\mathrm{Ag} / \mathrm{AgClO}_{4}$ as the reference electrode. The scan rate was $100 \mathrm{mVs}^{-1}$. The measurement was performed under nitrogen atmosphere. All potentials are referenced to the potential of ferrocene/ferrocenium cation couple.

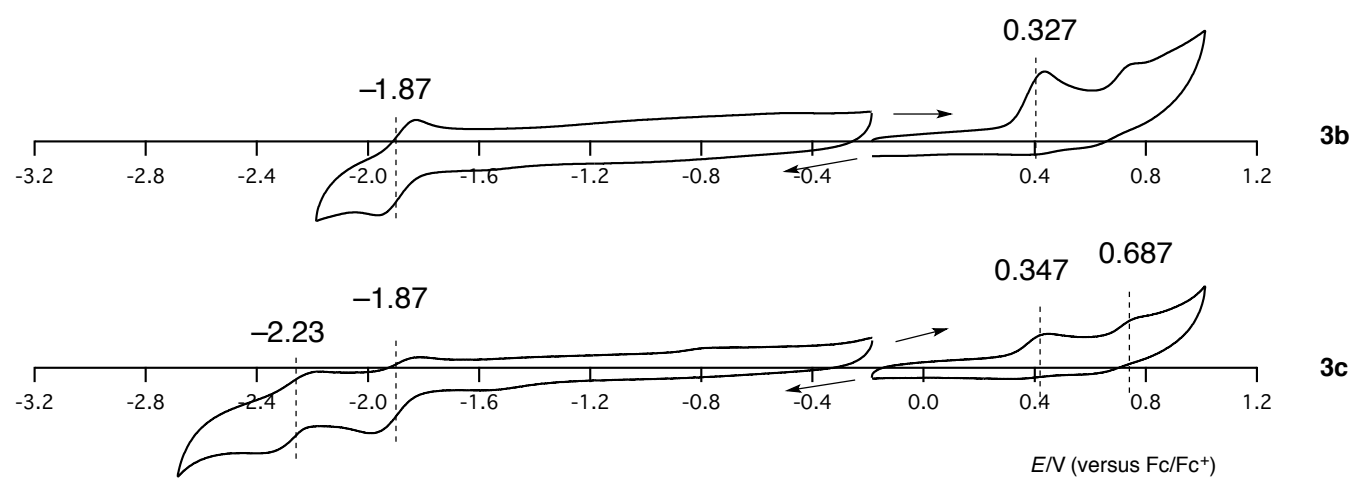

Figure S21. Cyclic voltammograms of $\mathbf{3 b}$ and $\mathbf{3 c}$.

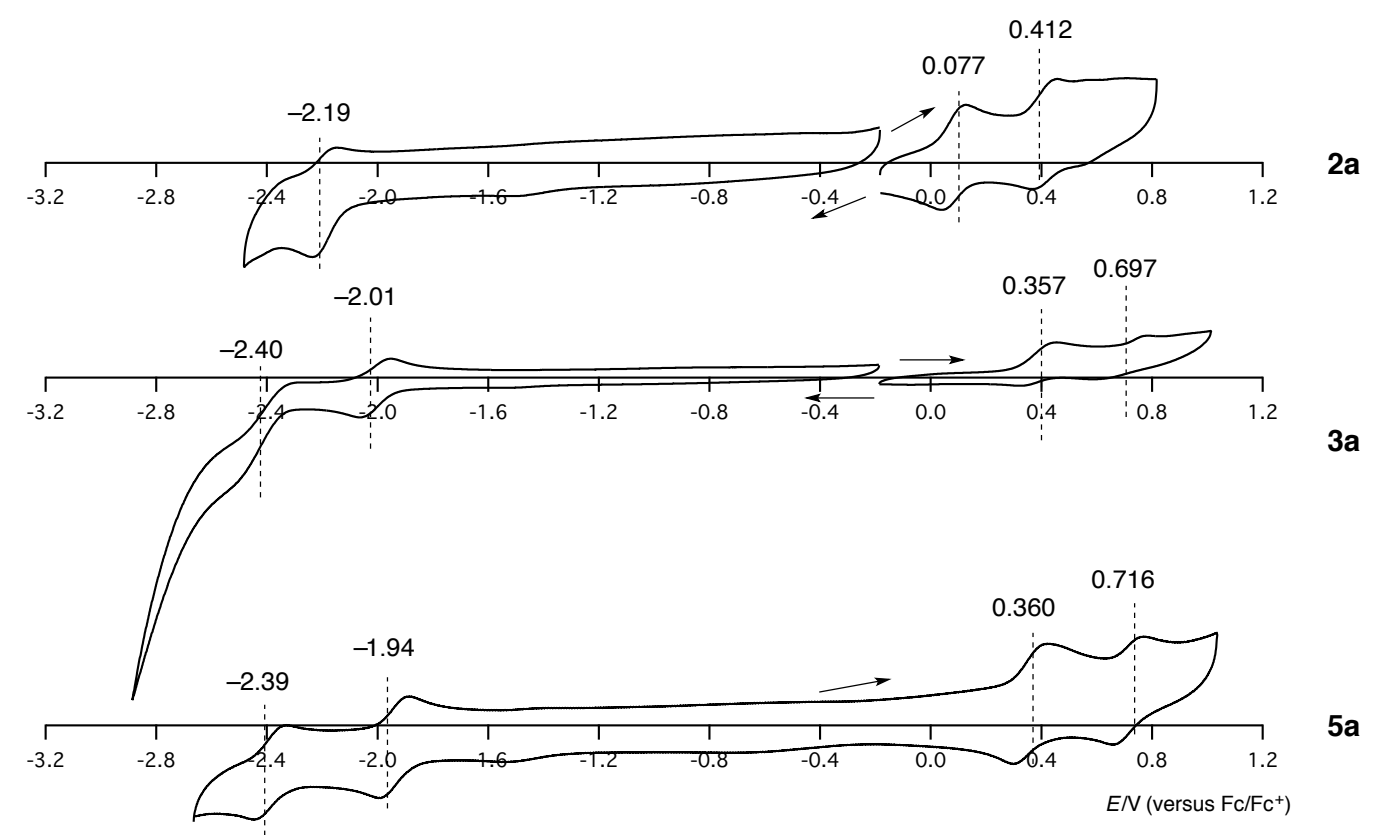

Figure S22. Cyclic voltammograms of $\mathbf{2 a}, \mathbf{3 a}$, and $\mathbf{5 a}$. 


\section{Theoretical calculations}

All calculations were performed using the Gaussian 09 program. ${ }^{1}$ The molecular orbitals of $2 \mathbf{a}$ and $5 \mathbf{a}$ were calculated based on the geometries of crystal structures by the DFT method using the CAM-B3LYP 2 functional and the 6-31G(d) basis set for C, H, N, and O atoms and LanL2DZ basis set for Zn or Ni metal. The oscillator strengths of 3a and 5a were calculated by the TD DFT method at the CAM-B3LYP/6-31G(d) level.
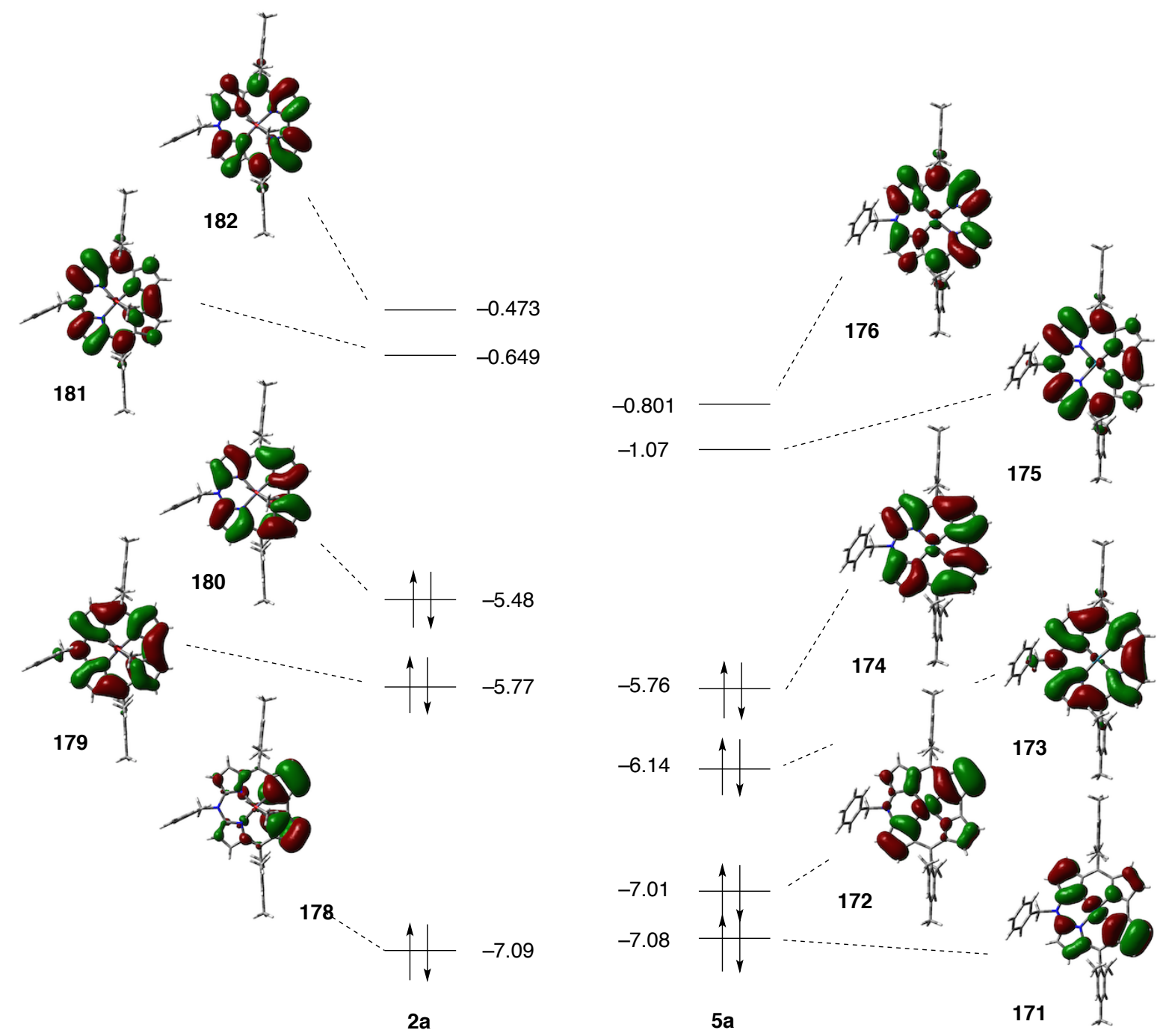

Figure S23. MO diagrams of $\mathbf{2 a}$ and $\mathbf{5 a}$. 


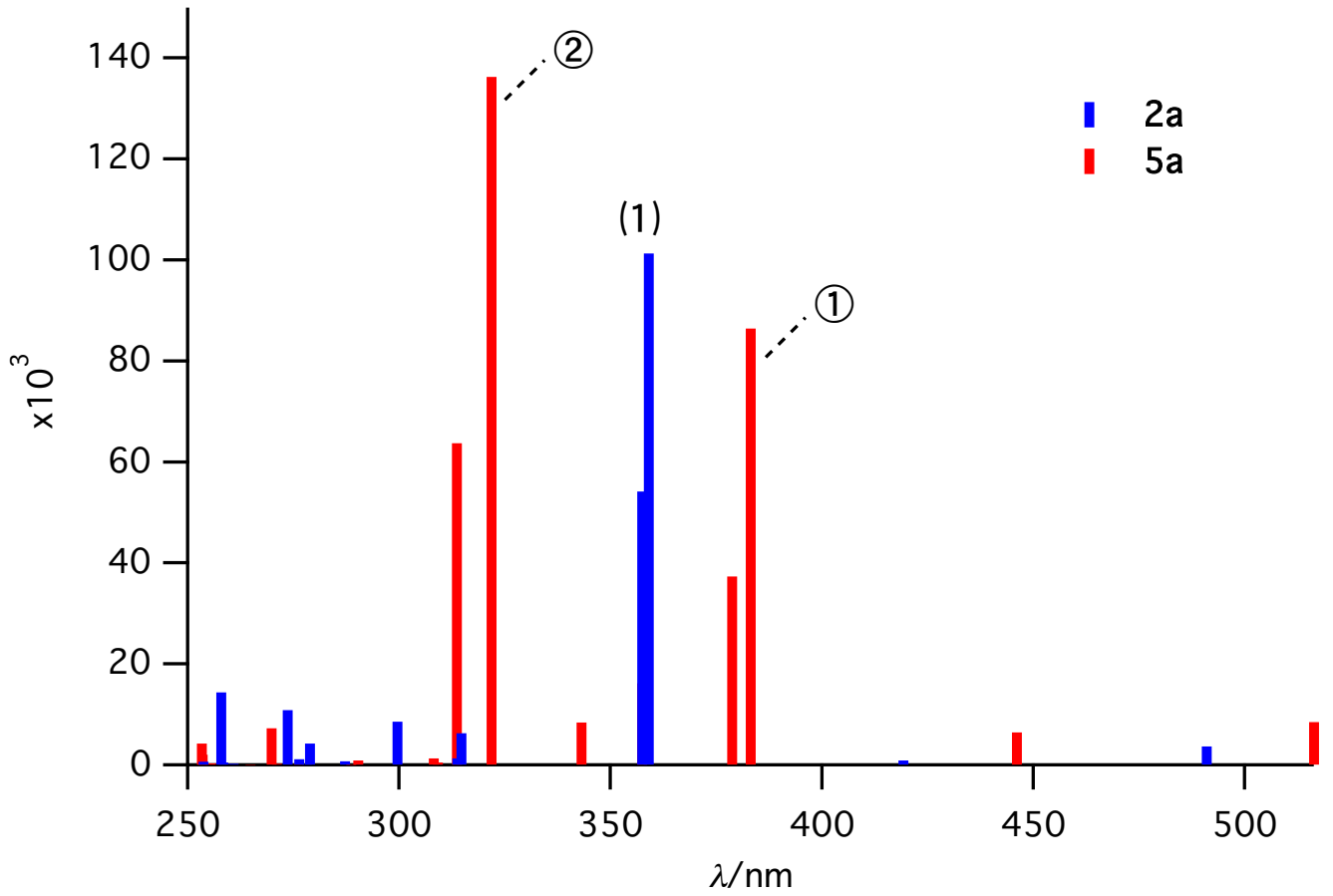

(1) $171->176 \quad-0.11121$

$172->175 \quad-0.28133$

$173 \rightarrow 175 \quad 0.56017$

$173 \rightarrow 176 \quad 0.16680$

$174->176 \quad-0.21231$

(2) $165 \rightarrow 175 \quad 0.10713$

$171->175 \quad 0.44557$

$172 \rightarrow 175 \quad-0.25483$

$172 \rightarrow 176 \quad 0.16828$

$173->176 \quad-0.34032$

$174->175 \quad-0.22379$

(1) $179->181 \quad 0.53945$

$180->182 \quad-0.44193$

Figure S24. Calculated oscillator strength of 2a (blue) and 5a (red). 


\section{References}

(1) Frisch, M. J.; Trucks, G. W.; Schlegel, H. B.; Scuseria, G. E.; Robb, M. A.; Cheeseman, J. R.; Scalmani,

G.; Barone, V.; Mennucci, B .; Petersson, G. A.; Nakatsuji, H.; Caricato, M.; Li, X.; Hratchian, H. P.;

Izmaylov, A. F.; Bloino, J.; Zheng, G.; Sonnenberg, J. L.; Hada, M.; Ehara, M.; Toyota, K.; Fukuda, R.;

Hasegawa, J.; Ishida, M.; Nakajima, T.; Honda, Y.; Kitao, O.; Nakai, H.; Vreven, T.; Montgomery, J. A., Jr.;

Peralta, J. E.; Ogliaro, F.; Bearpark, M.; Heyd, J. J.; Brothers, E.; Kudin, K. N.; Staroverov, V. N.; Kobayashi, R.; Normand, J.; Raghavachari, K.; Rendell, A.; Burant, J. C.; Iyengar, S. S.; Tomasi, J.; Cossi, M.; Rega, N.; Millam, J. M.; Klene, M.; Knox, J. E.; Cross, J. B.; Bakken, V.; Adamo, C.; Jaramillo, J.; Gomperts, R.;

Stratmann, R. E.; Yazyev, O.; Austin, A. J.; Cammi, R.; Pomelli, C.; Ochterski, J. W.; Martin, R. L.;

Morokuma, K.; Zakrzewski, V. G.; Voth, G. A.; Salvador, P.; Dannenberg, J. J.; Dapprich, S.; Daniels, A. D.;

Farkas, Ö.; Foresman, J. B.; Ortiz, J. V .; Cioslowski, J.; Fox, D. J. Gaussian, Inc., Wallingford CT, 2009.

(2) Yanai, T.; Tew, D.; Handy, N. Chem. Phys. Lett., 2004, 393, $51-57$. 